\title{
Alain TOURAINE
}

Sociologue, directeur d'études à l'Ecole des Hautes Etudes en Sciences Sociales

(1978)

\section{"Théorie et pratique d'une sociologie de l'action."}

\author{
Un document produit en version numérique par Jean-Marie Tremblay, bénévole, \\ professeur de sociologie au Cégep de Chicoutimi \\ Courriel: jean-marie tremblay@uqac.ca \\ Site web pédagogique : http://www.uqac.ca/jmt-sociologue/ \\ Dans le cadre de: "Les classiques des sciences sociales" \\ Une bibliothèque numérique fondée et dirigée par Jean-Marie Tremblay, \\ professeur de sociologie au Cégep de Chicoutimi \\ Site web: http://classiques.uqac.ca/ \\ Une collection développée en collaboration avec la Bibliothèque \\ Paul-Émile-Boulet de l'Université du Québec à Chicoutimi \\ Site web: http://bibliotheque.uqac.ca/
}




\section{Politique d'utilisation de la bibliothèque des Classiques}

Toute reproduction et rediffusion de nos fichiers est interdite, même avec la mention de leur provenance, sans l'autorisation formelle, écrite, du fondateur des Classiques des sciences sociales, Jean-Marie Tremblay, sociologue.

Les fichiers des Classiques des sciences sociales ne peuvent sans autorisation formelle:

- être hébergés (en fichier ou page web, en totalité ou en partie) sur un serveur autre que celui des Classiques.

- servir de base de travail à un autre fichier modifié ensuite par tout autre moyen (couleur, police, mise en page, extraits, support, etc...),

Les fichiers (.html, .doc, .pdf, .rtf, .jpg, .gif) disponibles sur le site Les Classiques des sciences sociales sont la propriété des Classiques des sciences sociales, un organisme à but non lucratif composé exclusivement de bénévoles.

Ils sont disponibles pour une utilisation intellectuelle et personnelle et, en aucun cas, commerciale. Toute utilisation à des fins commerciales des fichiers sur ce site est strictement interdite et toute rediffusion est également strictement interdite.

L'accès à notre travail est libre et gratuit à tous les utilisateurs. C'est notre mission.

Jean-Marie Tremblay, sociologue

Fondateur et Président-directeur général, LES CLASSIQUES DES SCIENCES SOCIALES. 
Cette édition électronique a été réalisée par Jean-Marie Tremblay, bénévole, professeur de sociologie au Cégep de Chicoutimi à partir de :

\section{Alain TOURAINE}

\section{“Théorie et pratique d'une sociologie de l'action.”}

Un article publié dans la revue Sociologie et sociétés, vol. $10, \mathrm{n}^{\circ} 2$, 1978, p. 149-188. Montréal : Les Presses de l’Université de Montréal.

Polices de caractères utilisée :

Pour le texte: Times New Roman, 14 points.

Pour les citations : Times New Roman, 12 points.

Pour les notes de bas de page : Times New Roman, 12 points.

Édition électronique réalisée avec le traitement de textes Microsoft Word 2008 pour Macintosh.

Mise en page sur papier format : LETTRE US, 8.5'’ x 11’’)

Édition numérique réalisée le 19 juin 2012 à Chicoutimi, Ville de Saguenay, Québec.

\section{Fait avec}




\section{Alain TOURAINE}

directeur d'études à l'Ecole des Hautes Etudes en Sciences Sociales

\section{"Théorie et pratique d'une sociologie de l'action.”}

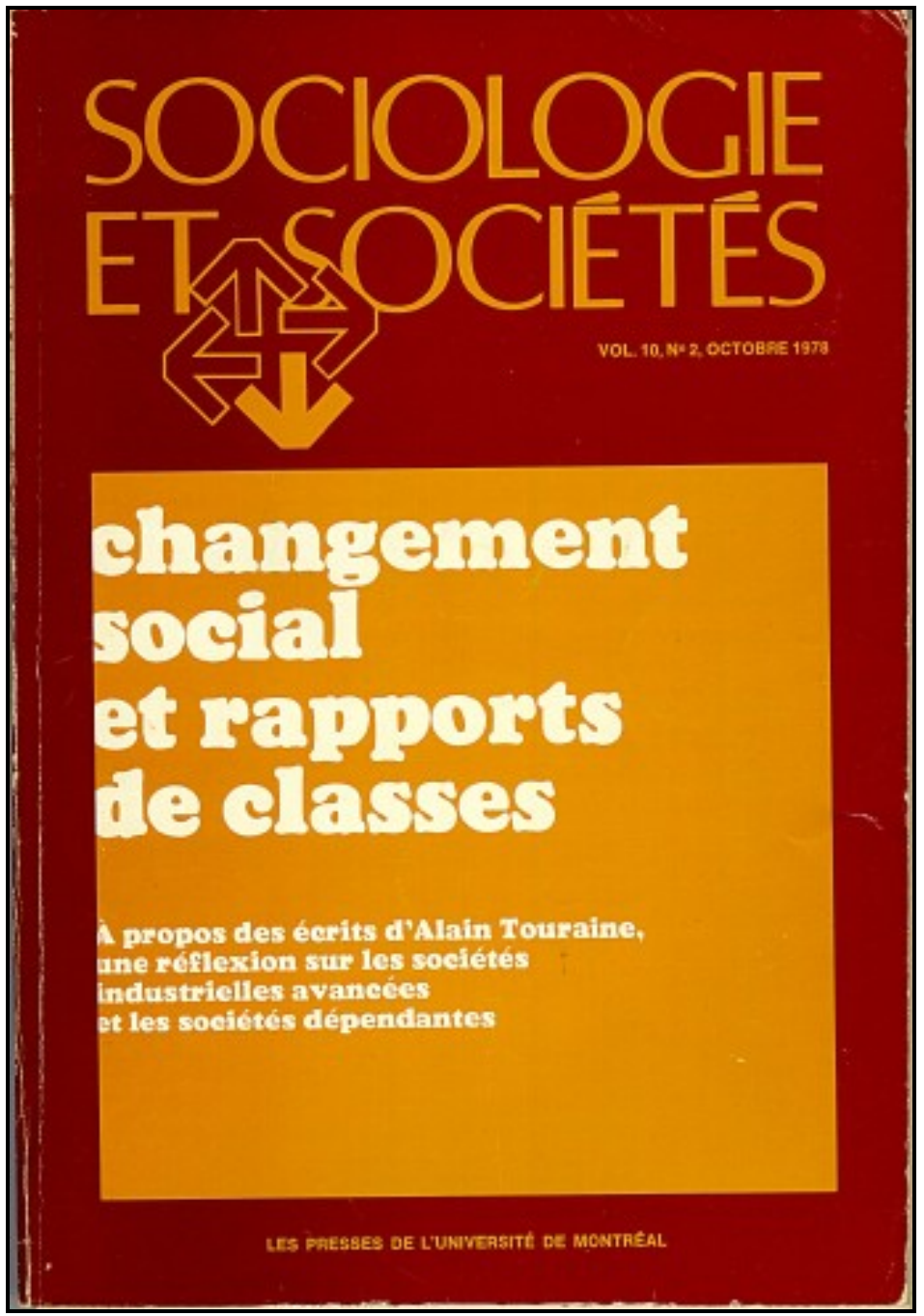

Un article publié dans la revue Sociologie et sociétés, vol. 10, n², 1978, p. 149-188. Montréal : Les Presses de l’Université de Montréal. 


\section{Table des matières}

$\underline{\text { Introduction }}$

I. De la société à l'action sociale

II. Les systèmes d'action sociale

III. L'analyse diachronique

Conclusion en forme d'introduction

RÉSUMÉ / SUMMARY / RESUMEN 


\section{Alain TOURAINE \\ “Théorie et pratique d'une sociologie de l'action.”}

Un article publié dans la revue Sociologie et sociétés, vol. $10, \mathrm{n}^{\circ} 2$, 1978, p. 149-188. Montréal : Les Presses de l’Université de Montréal.

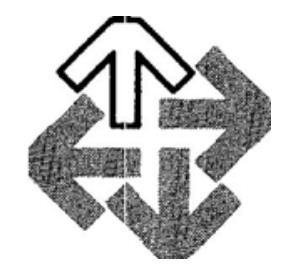

\section{INTRODUCTION}

$\underline{\text { Retour à la table des matières }}$

À la fin de ce colloque, après en avoir lu tous les textes et écouté les exposés et les discussions, je sens la tentation de revenir une fois encore à ce qui a été si souvent ma tâche la plus ingrate. Ne dois-je pas, en écoutant telle ou telle interprétation de certaines idées que j'ai pu élaborer au cours des vingt dernières années, veiller à ce que soit sauvegardé leur sens originel ? Non pas de manière magistrale, comme s'il s'agissait d'établir une orthodoxie, de défendre le territoire d'une théorie, alors que tous ceux qui étaient réunis à Bishop savent bien que nous n'avons pas discuté autour d'une théorie mais plus modestement et plus concrètement autour d'idées ; mais parce qu'il est indispensable assurément que ces idées se renforcent les unes les autres par la cohérence de la démarche où elles se placent et qui peut être menacée par des interprétations trop particulières de tel ou tel aspect de l'ensemble. Je n'éprouverais aucune difficulté à poursuivre aujourd'hui ce travail d'élaboration, de construction de l'échafaudage de 
notions e $\backslash$ de propositions qui doit permettre de redéfinir l'objet de l'analyse sociologique. Mais je crois que le temps n'est plus ii. ce travail de taupe et qu'après avoir creusé de longues galeries ramifiées, il faut revenir à la lumière et parler de manière plus pratique. L'insistance que j'ai mise pendant si longtemps à élaborer des idées, c'est-à-dire à préparer des recherches futures, ne provenait pas d'un choix libre ; j'étais forcé de m'enfermer dans cette réflexion solitaire et de tisser autour de moi un langage où [150] je savais bien que je risquais de m'enfermer mais qui me permettait de survivre, alors que le champ de la pensée sociale était tout entier occupé par des armées étrangères, je veux dire auxquelles je me sentais tout à fait étranger même si deci delà je pouvais trouver quelques bons côtés à leur domination. Je ne peux pas oublier en parlant aujourd'hui que j'ai toujours vécu à la fois très présent à mon époque et tout à fait isolé intellectuellement. À l'exception de quelques brèves années avant 1968 je n'ai jamais eu l'impression d'appartenir à un courant de pensée ou à une école et encore moins de penser de la même manière que des forces sociales ou politiques, voire plus modestement que des institutions universitaires. Pendant toute cette période, en même temps que ma réflexion politique se bornait à combattre l'inacceptable, en particulier pendant l'interminable période des dernières guerres menées par la France contre la décolonisation, je menais une œuvre solitaire, qui suscitait la méfiance des maîtres de l'Université comme de ceux qui se faisaient les idéologues de l'abondance, qui était regardée avec méfiance par ceux qui se réclamaient d'une science de l'histoire démontrée par les progrès et les victoires du monde socialiste et finalement rejetée avec agressivité ou avec mépris par les esprits sectaires et presque terroristes qui au lendemain de Mai 68 s'installèrent comme rats dans le fromage universitaire et cherchèrent à masquer la faiblesse de leurs titres par l'arrogance de leurs prétentions à représenter le sens de l'histoire. Je n'avais donc pas le choix : il fallait bien que je me replie sur un travail de reconstruction, travail nécessairement lent puisqu'il était solitaire et puisqu'il ne pouvait pas bénéficier d'un va-et-vient entre la théorie et la pratique, d'un dialogue au moins entre le sociologue et ceux qui exercent des responsabilités dans la gestion ou dans le changement de la société. Aujourd'hui les temps ont changé. Non pas que mon discours ait trouvé en dehors de lui-même les garants qui lui 
manquaient mais parce que les autres discours se sont écroulés et, comme leur force apparente venait de ce qu'ils étaient en réalité l'idéologie de groupes dominants ou aspirants à l'être, une fois ces maîtres ou petits maîtres en crise, ces pensées sociales ont perdu toute consistance et surtout toute autorité. Dans ces conditions-là, pourquoi continuer un travail souterrain et ne pas chercher ouvertement, comme tout le monde, mon chemin dans les ruines. En réalité mon espoir est plus haut. Je pense que le temps est venu pour les sociologues et pour tous ceux qui réfléchissent sur la société de s'apercevoir que disparaît sous leurs yeux ce qui fut l'objet de leurs études, la société, considérée comme système, structure ou pour parler plus simplement comme objet et qu'il fallait nommer ce qui depuis longtemps déjà aurait dû être la seule préoccupation des sociologues, à savoir l'action sociale et par conséquent les rapports sociaux qui n'en sont pas séparables. J'essaierai d'expliciter et de justifier cette idée mais il faut d'abord en tirer quelques conséquences pour l'orientation de ma réflexion présente. Je ne m'enfermerai plus dans la construction d'un système mais, le considérant provisoirement comme assez élaboré pour pouvoir être utilisé, je choisis à la fois de définir dans son unité une démarche et de l'autre côté de chercher comment elle peut conduire à l'élaboration d'une méthode, c'est-à-dire très concrètement d'un mode particulier de recueil et d'analyse d'informations pertinentes pour l'étude des problèmes posés. Ces priorités n'empêcheront pas que je doive ici ou là revenir sur des notions déjà proposées, mais chaque fois je m'efforcerai de le [151] faire non pas en faisant progresser la construction de principes d'analyse mais en faisant mieux apparaître ce qui relie chaque notion particulière à une inspiration d'ensemble et ce qui en elle appelle aussi l'emploi de nouvelles méthodes de recherche. 


\section{DE LA SOCIÉTÉ À L'ACTION SOCIALE}

$\underline{\text { Retour à la table des matières }}$

La rupture avec l'ancienne conception de la sociologie, définie comme l'étude : de la société, est imposée d'abord par la reconnaissance du fait que la sociologie qu'on peut nommer classique fut presque exclusivement l'étude d'un type particulier de société, situé précisément dans le temps et dans l'espace. Encor1e aujourd'hui n'appelons-nous pas sociologues ceux qui étudient les sociétés industrialisées occidentales tandis que nous nommons anthropologues ceux qui étudient les sociétés sans écriture ou plus largement les sociétés contemporaines pré-industrielles, africanistes ceux qui étudient les sociétés africaines en développement et même soviétologues ceux qui étudient l'Union soviétique ? Presque tous les grands fondateurs de la sociologie consacrèrent leur réflexion au phénomène historique exceptionnel qu'était la naissance en Europe occidentale de l'économie, de la société et de l'État qu'on peut nommer moderne. Weber en a recherché mieux que tout autre les conditions de formation, économiques et culturelles ; Durkheim s'est interrogé sur la manière de mettre fin aux grands déchirements de l'industrialisation et de la prolétarisation ; Marx, se définissant par rapport à tous ceux qui avant lui avaient insisté sur le rôle des classes sociales et de leurs conflits, déclara que son apport avait été de replacer l'analyse des classes dans l'étude de la structure économique de la société capitaliste moderne ; Tocqueville enfin fut sensible à la disparition des appartenances traditionnelles et au développement de la société de masse et des dangers qu'elle pouvait recéler. Pour quelle raison cette société industrielle capitaliste, pendant si longtemps seul exemple connu de société industrielle. suscita-t-el1e un discours sur elle-même qu'on appela la sociologie et qui considéra la société comme un objet quasi-naturel ? D'abord cette société ou plutôt les États nationaux qui la portaient disposa pendant longtemps d'une hégémonie mondiale quasi-complète. Son développement semblait dû entièrement à des facteurs internes. L'Angleterre victorienne put apparaître comme une société presque entièrement civile parce que la Royal Navy et les troupes coloniales" la flotte mar- 
chande et les banques transportaient au dehors la guerre et la conquête, les pré-conditions politiques de l'accumulation capitaliste. En deuxième lieu le développement industriel en Grande-Bretagne et dans une mesure moindre dans les pays voisins se réalisa sous la dictature de la bourgeoisie et put par conséquent être identifiée à la logique du profit capitaliste. Pendant longtemps il n'y eut presque pas de rencontres entre l'analyse des classes sociales et de l'exploitation capitaliste d'un côté et l'histoire de l'industrialisation de l'autre :. Les ouvriers subissaient l'exploitation mais leurs représentants ou ceux qui pouvaient parler en leur nom n'intervenaient pas, n'avaient pas le pouvoir d'intervenir dans le processus d'industrialisation. Cette situation ne se reproduisit pas en France, comme le nota très justement Marx. Dans ce pays où fut introduit le suffrage universel en 1848, forces sociales et agents politiques furent rarement en correspondance directe, comme le prouva l'élection de [152] Louis-Napoléon Bonaparte, qui ne recueillit pas les voix de la bourgeoisie bien qu'il fût destiné à devenir le meilleur agent politique du développement capitaliste en France. Mais il ne suffit pas d'analyser cette société industrielle classique avec les catégories qui furent les siennes; il faut aussi recourir aux nôtres. Si la société industrielle classique fut généralement conçue de manière positiviste, c'est-à-dire comme un ensemble de mécanismes, de règles et de pratiques cohérentes entre elles, organisées autour d'une logique centrale, définie elle-même en termes rationnels, en terme de recherche rationnelle d'intérêts économiques, c'est parce qu'elle présentait des caractères exceptionnels. Dans toutes les sociétés historiques, les pratiques économiques et sociales sont déterminées par l'ensemble d'orientations culturelles que je nomme l'historicité et en particulier par un modèle culturel qui fait appel à des garants métasociaux d'un ordre ou d'un autre. C'est ainsi que dans les sociétés agraires, les relations et les situations économiques et sociales sont replacées dans un ordre de significations qui est avant tout religieux. De la même manière dans les sociétés marchandes, les conduites économiques et sociales s'inscrivent dans un modèle culturel juridicopolitique, ce qui explique que la défense d'intérêts économiques prenne dans les conduites collectives la forme d'une lutte pour les libertés et pour la nation. Or la société industrielle et elle seule est commandée par un modèle culturel économique, par l'idée que le sens de l'histoire conduit vers la rationalité, l'instrumentalité, dont 
les conduites dites économiques sont la manifestations centrale. La société industrielle n'est pas différente des autres en ce que les conduites sociales y sont commandées par un type d'historicité et en particulier de modèle culturel mais elle est particulière en ceci que ce modèle culturel étant de nature historique et économique, cette société peut avoir l'illusion qu'elle n'est rien d'autre qu'un système de moyens rationnels au service de la rationalité des fins. Illusion positiviste démentie par les luttes sociales et par les crises économiques, par les destructions dans les pays colonisés comme par les mouvements de libération nationale, mais qui a commandé la représentation d'elle-même que la société industrielle a produite. Aujourd'hui encore elle aime se représenter comme la modernité opposée à la tradition, comme l' «achievement» opposé à l'« ascription », comme la production opposée à la reproduction, comme la forme la plus avancée d'une évolution à la fois historique et naturelle qui conduit de la solidarité mécanique à la solidarité organique, d'une faible à une forte différenciation, d'une capacité de changement limitée à une capacité presque illimitée. Aujourd'hui encore vit activement une pensée libérale qui défend, comme le fit en son temps Voltaire, tout ce qui détruit les préjugés, les coutumes et les traditions, qui augmente les choix et l'indétermination, qui permet donc le triomphe du calcul rationnel. Mais nous ne pouvons plus être dupes aujourd'hui de cette image de la société industrielle classique qui l'identifiait au sens de l'histoire ou plutôt nous devons reconnaître dans ce sens de l'histoire le garant métasocial propre à la société industrielle. Qu'il se présente sous la figure de la rationalisation et de la sécularisation n'empêche pas qu'il soit de même nature que les modèles culturels antérieurs au lieu d'être, comme il le croit lui-même, la suppression de toute transcendance et le triomphe de l'éthique de la responsabilité.

C'est certainement parce que les sociétés industrielles capitalistes centrales ont perdu leur hégémonie et parce que nous devons étudier des sociétés qui [153] dans leur immense majorité sont différentes de ce modèle classique que nous ne pouvons plus accepter aujourd'hui l'image de la société qui fut l'idéologie particulière des premières sociétés industrielles. 
En premier lieu l'identification de la société toute entière à la rationalité ou au moins au mouvement de rationalisation, qui fut l'idéologie dominante de la société américaine au moment de sa plus grande puissance et qui trouva son expression la plus élaborée dans la doctrine parsonienne, a été brutalement détruite par les crises qui ont atteint cette puissance et cette bonne conscience. D'un ,côté se sont développés, d'abord dans des milieux très limités puis dans des catégories de plus en plus larges de la population, des genres de vie et des idées en contradiction profonde avec les valeurs de la société industrielle. D'autre part, la guerre du Viet-Nam et d'autres expéditions analogues ont fait douter les plus timides de l'identification d'une société nationale avec la raison. Sur un plan beaucoup plus vaste, la scène mondiale a été de plus en plus occupée par la multiplicité des modes de développement nationaux ou régionaux. Il semblait acceptable au siècle dernier de penser que le personnage principal de l'histoire de l'Angleterre était la livre sterling ou au moins la City ; il est difficile de ne pas voir en notre siècle que les mouvements, les programmes et les volontés de développement, de transformation historique volontaire, l'emportent presque partout sur ce qu'on nomme d'un terme désuet les forces économiques. En Europe même au cours de l'industrialisation, le problème social principal fut celui de la misère ouvrière, c'est-à-dire de la subordination des travailleurs à l'accumulation du capital ; qui nierait qu'en notre siècle et dans cette partie du monde le problème qui trouble le plus les esprits et les consciences est celui du totalitarisme et en particulier des camps de concentration et de la torture. L'Allemagne hitlérienne et l'Union soviétique stalinienne et post-stalinienne sont marquées à jamais par les images horribles de la répression mais la France a aussi créé des camps de concentration pour les réfugiés espagnols et a organisé la torture en Algérie. Au delà de nos frontières continentales, nous ne voyons presque que des entreprises étatiques, comme si les sociétés disparaissaient derrière les États. Comment dans de telles conditions continuer à confondre le fonctionnement de la société et le changement historique, comment croire qu'il existe des lois de développement historique inscrites dans une structure économique ? Dans les sociétés occidentales, qui peut encore penser, sauf quelques esprits paresseusement dogmatiques, que l'État n'est que l'agent d'une classe dominante, voire même de sa frac- 
tion centrale ; qui peut oublier l'énorme proportion du revenu national qui est redistribuée par des institutions politiques ; qui peut ne pas s'apercevoir que les agriculteurs, les fonctionnaires et parfois même des travailleurs industriels ont obtenu un accès à l'influence politique assez important pour que le gouvernement ne puisse plus apparaître comme la simple transcription politique de la logique du profit ? Enfin, puisque nous vivons ce qu'on appelle encore une crise économique, croit-on qu'on la comprenne en faisant appel seulement à des mouvements cycliques ou aux conditions de la formation du profit, en oubliant qu'elle est inséparable d'une profonde transformation des relations économiques mondiales et de nouvelles transformations technologiques. Il est devenu aussi difficile de réduire notre société à la logique de la classe dirigeante que de l'identifier au progrès de la productivité, de la rationalité et de l'abondance. [154] C'est pourquoi au cours de la dernière décennie, les courants de pensée d'inspiration marxiste se sont profondément transformés. Au lieu de réduire la société à la logique du profit capitaliste et d'affirmer ainsi le primat d'une infrastructure, ils ont redonné la priorité à la logique globale d'un mode de production c'est-à-dire à l'ordre social tel qu'il est établi et maintenu par l'État et par ce qu'on a de plus en plus souvent appelé ses appareils idéologiques. Ce qui a conduit à une étrange situation. Au moment même où des sociétés industrielles connaissaient une croissance unique dans l'histoire, où elles vivaient donc des transformations économiques, sociales et culturelles exceptionnelles, l'image de la société qui recueillit l'appui du plus grand nombre d'intellectuels fut celle d'une société de reproduction et d'enfermement, imposant sa logique multiforme à une masse déstructurée, à une plèbe inorganisée. Production doctrinale qui ne témoigne que de l'extraordinaire séparation du milieu universitaire et de la société où il vit isolé mais qui est si éloignée de la réalité qu'elle ne peut produire d'études concrètes que dans des domaines extrêmement limités. L'école par exemple est présentée surtout comme un instrument de reproduction sociale alors qu'elle est traversée par des transformations fondamentales, d'un côté un accès massif à l'éducation et de l'autre une association de plus en plus directe du niveau d'études et des chances de carrière ou de revenu. Ainsi semble se terminer une longue époque. D'un côté l'orgueil de la sociologie parsonienne a été remplacé par les observations 
désabusées et parfois cyniques des interactionnistes symboliques et des ethno-méthodologues. On ne croit plus aux valeurs mais on reconnaît que la société est une comédie dont les acteurs respectent les règles. De l'autre l'idée de la dictature de la bourgeoisie et du profit, qui laissait au moins une grande place aux luttes de classes voire à des efforts d'institutionnalisation est remplacée par l'image d'une société-ordre, imposant de plus en plus étroitement la conformité et enfermant toutes les catégories de déviants. Double forme de dégénérescence de la sociologie classique, fonctionnaliste d'un côté, marxiste de l'autre. Décadence générale si prononcée qu'on peut se demander aujourd'hui s'il faut encore parler de sociologie ou s'il ne convient pas plutôt d'abandonner ce mot qui fut si étroitement lié à l'image de soi qu'inventa un certain type de société. Le doute qui envahit les esprits porte aujourd'hui sur l'utilité d'une analyse des systèmes sociaux. Ne faut-il pas redonner la priorité à l'étude du changement, du développement, c'est-àdire aussi de la volonté des États plutôt que de la logique des intérêts. Je me refuse à de tels abandons mais je me refuse plus encore à maintenir une image de la société qui ne peut plus conduire à aucune pratique de recherche, qui se dégrade en discours doctrinal ou, comme on le dit prétentieusement, en théorie. Il faut pour le moins rejeter les trois idées sur lesquelles a reposé la sociologie classique. On ne peut confondre structure et changement; il faut renoncer à toute conception évolutionniste. En deuxième lieu il est devenu impossible d'admettre que l'action est subordonnée à des formes objectives d'organisation sociale, impossible de croire que l'action n'est que l'événement qui flotte à la surface des structures profondes de l'économie. En dernier lieu il faut revenir à la reconnaissance du fait qu'une société n'est pas un ensemble de pratiques, n'est pas la mise en œuvre d'une tendance ou d'un principe de rationalité, n'est pas susceptible d'être expliquée par une histoire naturelle, mais doit être conçue comme le résultat d'actions sociales, d'interventions [155] d'acteurs et de collectivités sur euxmêmes. En un mot, il faut renoncer à l'idée que les conduites sont déterminées par des situations et reconnaître que ce qu'on nomme situation n'est que le produit fragile et provisoire de rapports sociaux, de relations politiques et d'orientations culturelles. 
Avant d'entrer à nouveau dans la construction d'une analyse sociologique, il faut donc se demander directement ce que signifie aujourd'hui être sociologue ; pourquoi défend-on aujourd'hui l'idée que la société est un réseau de rapports et d'acteurs sociaux et' à quoi cette conception s'oppose+ elle ? Elle s'oppose d'abord, je l'ai déjà dit, à la réduction de l'analyse sociale à celle du changement, c'est-à-dire l'analyse politique et même plus précisément à l'étude des politiques des États. Cette position ne se justifie pas seulement pour des raisons intellectuelles. Elle repose aussi sur des choix dont il faut être conscient. Refuser de laisser libre champ à l'analyse de l'État, c'est refuser d'abord de reconnaître les pleins pouvoirs à l'État lui-même. Et n'est-ce pas notre principal souci aujourd'hui ? Ne sommes-nous pas angoissés de voir l'État ou les forces étatiques détruire, remplacer ou masquer les forces sociales et par conséquent l'action sociale elle-même et ses orientations culturelles autant que les conflits sociaux où elle est toujours engagée ? J'y reviendrai plus loin tant le sujet est d'une importance primordiale. Je dis ici que le propre de ceux qu'on nomme sociologues et qui appartiennent dans leur immense majorité aux sociétés qui furent et qui sont encore les plus civiles, est d'affirmer la priorité des acteurs et des enjeux sociaux sur les contraintes et les volontés des États. Il s'agit de refuser la mise entre parenthèses de : la société qui caractérise tous les régimes et toutes les tentatives totalitaires. Non pas seulement pour des raisons morales mais parce que nous savons bien que cette société devenue invisible ne disparaît jamais et qu'elle est seulement réprimée. Telle est la leçon que viennent de nous donner et que nous donneront longtemps encore les dissidents soviétiques, et d'abord Soljenitsyne. Le plus extraordinaire dans leur action n'a pas été la dénonciation du pouvoir et de ses crimes mais la preuve apportée que derrière la façade militarisée de l'État, la vie sociale continuait à exister, l'analyse, l'espoir et la lutte à survivre malgré les interdictions et les condamnations. Nos joies les plus grandes ont été ces dernières années de voir réapparaître des sociétés que l'on croyait à jamais disparues derrières les murailles d'Un régime totalitaire. À l'autre bout de l'Europe, l'Espagne a réapparu comme société. Là où on attendait la rupture, la violence, l'affrontement d'un État et d'un contre-État, voici que surgit l'innovation, le mouvement social, le débat politique et le bond en avant des libertés. Du côté soviéti- 
que, c'est la répression qui l'emporte encore mais l'essentiel a déjà été fait ; nous savons désormais que l'État ment lorsqu'il parle au nom de la société, que le silence était contraint et que la contestation n'a jamais disparu même au prix des sacrifices les plus grands. Ainsi peu à peu, après un quart de siècle dominé par la montée des États, apercevons-nous quelques terres qu'on croyait englouties à jamais et que laisse il nouveau apercevoir le flux qui semble maintenant descendant du pouvoir absolu des États. Du côté des sociétés capitalistes industrialisées, les difficultés et les adversaires sont d'un autre ordre. Après un long siècle d'illusion dl l'objectivité sociale, il semble que nous entrions dans une culture qui est dominée plus encore que la précédente par des critères positifs de jugement. [156] Dans la mesure même où disparait le sacré et là où il n'est pas remplacé par le pouvoir absolu de l'État, il semble ne laisser la place qu'à la recherche du plaisir considéré comme l'équivalent du rendement dans l'ordre de la consommation. Ainsi semble se défaire la vie sociale, simple moyen de parvenir à la satisfaction des besoins, à la jouissance du corps, à une liberté indéterminée et qui ne peut plus fonder de rapports sociaux autres que les circonstances. Au risque de paraître tenir un discours archaïque, je dois dire que la sociologie à laquelle je tiens me semble être d'abord une défense contre cette société de consommation. Pour des raisons qui ne sont probablement pas toutes rationnelles, je considère cette exaltation présente de la consommation comme suicidaire. Non seulement elle conduit nos sociétés dans leur ensemble et la plupart de leurs membres à se replier sur des objectifs immédiats comme si leur avenir était assuré, comme si elles n'avaient pas de concurrence extérieure, comme s'il leur revenait par décret divin de maîtriser les nouvelles techniques, c'est-à-dire de réussir les grands investissements qui commandent le développement de ces techniques et des conditions sociales qui permettent leur développement. Pendant les décennies récentes, en dehors du petit monde intellectuel enfermé dans l'idée que la société est un système de reproduction, la majorité de nos contemporains s'est laissée emporter par cet immense élargissement du marché. Les intérêts des marchands l'ont si complètement emporté qu'ils ont fait oublier aussi bien les problèmes de la production que ceux de la justice sociale. C'est en ce sens que nos sociétés, tout en étant les plus riches et 
les plus libres, portent en elles la marque de la décadence. Maintenant que nous sommes entrés dans ce qu'on nomme la crise, on va s'apercevoir assez vite que la dégringolade peut être rapide si on ne prend pas les mesures nécessaires pour redonner à l'avenir la priorité qu'il doit avoir sur le présent et d'abord pour être capable de faire basculer notre consommation des produits industriels vers les produits post-industriels, ce qui est une opération aussi difficile que le fut celle du transfert des ressources du domaine agricole et artisanal vers le domaine industriel. Si j'ai placé l'idée d'historicité au centre de la sociologie, c'est parce que je crois que toute société est gouvernée par autre chose qu'un principe de plaisir, qu'elle est construite à partir du travail qu'elle exerce sur ellemême. Ce qui m'amène à dire, pour conclure cette partie d'introduction, que la sociologie de l'action telle que je la défends est la seule qui soit entièrement matérialiste, parce qu'elle est la seule qui explique l'ensemble des pratiques sociales à partir de la production de la société par elle-même, à partir de son travail sur elle-même. Travail qui n'est pas une activité mais un ensemble d'orientations normatives qui commandent les formes d'organisation sociales et les conduites collectives ou individuelles. Rien n'est plus faux que de voir dans cette sociologie de l'action une nouvelle version d'une philosophie du sujet; elle est tout le contraire puisque ce que je nommais dans mes premiers livres le sujet historique n'est que l'acteur social dans la mesure où il participe directement et conftictuellement à ses orientations culturelles qui tracent le champ de l'action sociale. En résumé la sociologie de l'action s'écarte des illusions objectivistes propres à l'époque industrielle et s'efforce de construire l'ensemble de l'organisation sociale et culturelle à partir de la connaissance du mode de production de la société par elle-même. Ceci l'amène à combattre contre toutes les formes de [157] subordination de la société à l'État et à son projet de transformation historique et à combattre aussi contre la réduction de plus en plus dérisoire de la vie sociale à l'acquisition, au plaisir et à l'échange.

J'ai tenu à situer ma réflexion avant d'en présenter les orientations principales, car je crois qu'il ne faut pas séparer une connaissance dite scientifique d'une pensée sociale dont on reconnaîtrait la charge idéologique ou doctrinale. Notre travail doit être conscient de sa double 
nature : discours interprétatif d'une société sur elle-même et connaissance qui se forme dans la mesure même où notre société peut enfin se concevoir comme le produit de sa propre action et non plus comme la manifestation d'une nature sociale.

\section{LES SYSTÈMES D'ACTION SOCIALE}

Retour à la table des matières

Si on considère la société comme un objet naturel elle apparaît à celui qui veut expliquer les conduites sociales comme une situation, comme un ensemble de déterminants, ce qui conduit à résumer l'explication sociologique par la formule : la situation explique la conduite. Or la sociologie classique a constamment réagi contre cette conception superficielle. La célébrité de Durkheim ne vient-elle pas en grande partie de ce que, pour expliquer le suicide, au lieu de considérer cette conduite comme déterminée par des facteurs démographiques ou économiques, il l'a analysée comme la manifestation d'une rupture ou d'une désorganisation des relations sociales ? Ainsi se trouve définie la méthode sociologique. Elle consiste à expliquer les conduites des acteurs par les relations sociales dans lesquelles ils sont placés. Ceci justifie l'importance prise par la théorie des rôles puisque le rôle peut être défini par l'ensemble des attentes légitimes du partenaire à l'égard de l'acteur dans la relation sociale qui les unit. Mais il reste alors à définir ce qu'est une relation sociale afin de montrer clairement le lien essentiel qui existe entre une sociologie de l'action et la définition des relations sociales comme objet de l'analyse sociologique.

Une relation sociale est une interaction placée dans un champ. Ceci oppose les relations sociales aux relations non sociales ou intersociales. Si deux adversaires combattent pour la possession d'un territoire, si deux partenaires s'opposent dans un jeu à somme nulle, il n'existe pas entre ces acteurs de relation sociale. Pour que celle-ci apparaisse, il faut que les deux acteurs appartiennent au même ensemble, se réfèrent en particulier aux mêmes normes et à un minimum de règles institutionnelles. Les relations collectives de travail sont en partie sociales 
et en partie non sociales. Elles sont quelquefois une forme atténuée de guerre civile ; elles deviennent des relations sociales à partir du moment où elles sont de quelque manière reconnues et institutionnalisées. Cette distinction ne vise pas à sous-estimer l'importance des relations intersociales, relations de marché, de concurrence, de guerre. Bien au contraire. Mais on verra qu'elles représentent au cœur même du champ des relations sociales une réalité bien différente, les conduites de changement historique.

Que faut-il entendre par champ ? La première définition qui vient d'être donnée est manifestement insuffisante. Les acteurs ne sont pas dans un champ, [158] ce qui reviendrait à considérer celui-ci comme une situation définie indépendamment des acteurs qui y sont placés. Un champ est construit par une intervention sociale sur l'organisation sociale et les acteurs sont définis par leur position différentielle par rapport à cette intervention. Un exemple simple fera mieux comprendre cette formule apparemment abstraite. La relation d'un ouvrier et d'un contremaître dans une entreprise n'est pas définie, malgré les apparences, par les règles d'organisation de cette entreprise, comme si celle-ci définissait les droits et les devoirs de chacun, du contremaître autant que de l'ouvrier. L'insuffisance de cette formule apparaît à l'évidence si on remplace contremaître par directeur général. Chacun sent que le directeur général n'est pas soumis aux règles de fonctionnement de l'entreprise au même titre que l'ouvrier puisque le propre d'une direction est d'établir et de faire respecter ces règles, même si celles-ci sont dans une certaine mesure négociées. La relation de l'ouvrier et du contremaître est définie par un système d'autorité et celui-ci à son tour est imposé à une organisation par un pouvoir dont le fondement est extérieur à cette organisation, qu'il s'agisse de la propriété des moyens de production ou du pouvoir politique. Le contremaître est celui qui exerce l'autorité directe sur l'ouvrier ou qui est censé le faire. L'ouvrier est défini comme celui qui ne participe pas à l'autorité, qui n'a donc pas accès à l'influence, qui ne détient pas le principe du pouvoir. La relation entre l'ouvrier et le contremaître est déterminée par les rapports de classes et par ce que je nomme les rapports institutionnels, c'est-à-dire par l'influence comparée des divers acteurs sur la prise des décisions considérées comme légitimes. Toute relation sociale est donc inégalitaire et 
comporte une dimension de pouvoir. Telle est la proposition la plus élémentaire de la sociologie. Elle n'est pas sans conséquences à notre époque où reçoit tellement d'importance la recherche de la spécificité et de la différence. Qu'une catégorie sociale comme des femmes ou un groupe ethnique ou les homosexuels, réclame la reconnaissance de ses particularités, de son identité, ne peut avoir aucun sens pour le sociologue. De telles revendications peuvent recouvrir des changements culturels importants ; elles sont entièrement indéterminées d'un point de vue social, ce qui conduit soit à les considérer comme marginales soit au contraire à y voir l'expression des intérêts de groupes dominants car c'est le propre des revendications de tels groupes de se présenter de manière voltairienne en combattant les barrières, les préjugés et les traditions au nom d'une liberté qui reste socialement indéterminée. Au contraire une revendication est socialement importante dans la mesure où elle met en cause une relation sociale précise et par conséquent le pouvoir exercé par un des termes de la relation sur l'autre. Toute relation sociale est une relation de pouvoir alors qu'on peut imaginer aisément une relation intersociale entre deux partenaires égaux ou au moins non liés l'un à l'autre par une relation de pouvoir. Tel est le cas de l'affrontement politico-militaire entre l'empire américain et l'empire soviétique par exemple. Il reste, pour établir une liaison complète entre la définition de la sociologie comme étude de l'action et la proposition que les relations sociales constituent son objet spécifique, à définir les divers modes ou niveaux d'intervention de la société sur elle-même ou, si on préfère, les différents niveaux de formation du pouvoir. Si on part, comme dans l'exemple déjà utilisé, du niveau de l'organisation sociale, c'est-àdire de la coopération d'acteurs en vue [159] d'un but défini par un certain rapport de la collectivité et de son environnement, donc par un certain travail, l'autorité qui définit les rôles et les relations de rôles dans cette organisation repose sur des décisions institutionnelles, c'est-à-dire sur des mécanismes politiques, ce qui constitue le deuxième niveau de la vie sociale, un deuxième type de système d'action sociale. Mais les relations politiques sont elles-mêmes situées à l'intérieur d'un champ. Elles ne peuvent pas dépasser certaines limites. Ce champ est parfois dessiné très nettement par une constitution, par des lois fondamentales. Ses limites sont établies par un pouvoir qui est celui d'une classe dirigeante, c'est-à-dire de 
l'acteur qui tente de s'approprier les moyens par lesquels une société agit sur elle-même et de s'identifier à ces moyens, c'est-à-dire au fondement de la normativité. Ceci définit un troisième système d'action sociale que je nomme champ d'historicité. Il est composé par les rapports de classes qui sont eux-mêmes des rapports de pouvoir définis par une relation conflictuelle à l'historicité, c'est-àdire au mode de production de la société par elle-même.

$\mathrm{n}$ faut maintenant rappeler ce qu'est l'historicité. J'ai dit maintes fois qu'elle était l'ensemble des formes de travail de la société sur elle-même, d'un mode de connaissance par lequel est constitué un rapport de la société à son environnement, d'un mode d'investissement par lequel la société constitue son activité économique, et enfin d'un modèle culturel par lequel elle se représente sa propre créativité, sa propre capacité d'action sur elle-même, ce qui fonde le champ de l'éthique. Mais l'essentiel n'est-il pas de préciser les relations entre ces trois composantes et surtout de se demander d'où elles viennent afin d'écarter le risque d'un idéalisme élémentaire, selon lequel les idées détermineraient les pratiques, idéalisme qui se heurte toujours à la question élémentaire : mais d'où ces idées viennent-elles? Il faut donc dire nettement que l'état de l'historicité et donc son contenu ne peuvent être définis que par un certain niveau d'intervention de la société sur elle-même, par la capacité de la société de produire son organisation et ses pratiques. C'est pourquoi l'élément central de l'historicité est l'investissement. Ce qui semble donner un primat à un facteur économique. Mais une telle formulation est plus dangereuse qu'utile car l'avantage reconnu au rôle de l'investissement nous conduit en sens tout à fait inverse de l'idée que les conduites s'expliquent par des déterminants économiques car l'investissement signifie la capacité d'intervenir, à l'aide de la partie non consommée du produit, sur l'ensemble de l'organisation économique pour créer ce qu'on peut nommer un mode de production ou plus précisément un mode de production économique de la société par elle-même. La forme la plus simple de cette analyse distingue quatre niveaux d'intervention de la société sur son activité économique. Certaines sociétés ne peuvent intervenir que sur la consommation et sur la reproduction de la force de travail. Les sociétés agraires traditionnelles n'investissent qu'en transformant une partie de la récolte en se- 
mence, qu'en consacrant une autre partie de cette récolte à la nourriture des enfants ou encore en consacrant des ressources à l'entretien des enfants ou encore en consacrant des ressources à l'entretien de guerriers capables de conquérir des terres ou des esclaves. Les sociétés marchandes sont celles qui appliquent l'investissement non plus seulement à ce domaine de la consommation mais à celui de la distribution des biens et services. Ces investissements portent [160] sur les routes, les transports en général, la création, la garantie et le transfert des monnaies, sur l'établissement de règles de droit et de moyens de les faire respecter. La société industrielle est celle qui ajoute à ces investissements de type agraire et commercial un nouveau type d'intervention économique de la société sur elle-même. Elle intervient sur l'organisation du travail. La société industrielle n'est pas définie par l'emploi de machines ou même de sources nouvelles d'énergie ; elle l'est par l'utilisation du capital pour la création de nouvelles formes d'organisation du travail qui introduisent la spécialisation non pas seulement au niveau des centres de production mais au niveau des ouvriers et des ateliers eux-mêmes. Enfin on peut nommer société programmée ou de manière plus vague et provisoire post-industrielle la société qui utilise le capital pour transformer non plus la consommation, la distribution et l'organisation du travail mais en plus la production elle-même, à la fois en introduisant des méthodes de gestion et en inventant des produits nouveaux, deux innovations qui ont en commun d'intervenir directement au niveau de la connaissance et donc d'ensembles complexes d'informations et de communications. Plus on s'élève du niveau le plus élémentaire d'investissement au niveau le plus élevé, plus s'élargit la capacité d'intervention économique de la société sur elle-même et plus aussi la société se conçoit comme étant produite par son propre travail. À l'opposé, dans les sociétés agraires traditionnelles, cette créativité n'est pas conçue comme étant l'attribut de la société mais comme ayant une origine métasociale. Le domaine social apparaît comme celui de la reproduction ou de mouvements cycliques tandis que la création est attribuée à un ordre qu'on peut appeler divin ou plus généralement religieux. Les sociétés marchandes ont créé une image de la créativité, ce que je nomme un modèle culturel, qui s'éloigne du modèle religieux tout en restant encore éloigné de l'idée de responsabilité de la société sur elle-même. L'image de la 
créativité est celle de la loi ou du Prince qui établit l'ordre et la civilisation en imposant des contraintes et des règles à la nature et en particulier aux passions humaines. La société industrielle est orientée par un modèle culturel profondément différent. Parce que cette société agit sur l'organisation du travail, elle se représente la créativité non plus comme un ordre mais comme un mouvement naturel qui conduit du simple au complexe, des systèmes indifférenciés aux systèmes fortement différenciés. Ce modèle culturel est donc évolutionniste et naturaliste. Il est impossible de concevoir un mode d'investissement entièrement séparé d'un modèle culturel, des investisseurs qui ne soient conduits que par la recherche d'avantages à l'intérieur d'un type particulier d'organisation sociale. Il faut se placer ici dans une tradition wéberienne et reconnaître que l'investissement est une action, est donc orienté par des valeurs culturelles et ne peut être réduit à un calcul rationnel. Cette association d'un mode d'investissement et d'un modèle culturel donne naissance à un mode de connaissance, à un travail pratique de connaissance sur la nature, qu'on pourrait fort bien appeler force de production dans la mesure où cette construction d'un modèle de nature commande directement la présence ou l'absence d'activités technologiques. On passe ainsi de l'idée que la société humaine et l'homme sont à l'image de l'univers, microcosmes insérés dans le macrocosme, à une autre représentation du monde dans laquelle celui-ci apparaît comme gouverné par des lois et des relations causales. Dans la société [161] industrielle, ces lois deviennent des lois d'évolution et la connaissance historique s'applique à la plupart des domaines. Enfin, les sociétés qui ont une capacité quasi illimitée d'action sur elles-mêmes, qui se considèrent comme responsables d'elles-mêmes ne font plus appel à un ordre de causes qui commanderait l'ordre des effets mais étudient les rapports d'interdépendance entre les phénomènes, élaborant ainsi une conception systémique de la nature. Ainsi les trois composantes de l'historicité sont-elles liées les unes aux autres en ce qu'elles sont les diverses composantes du travail de la société sur elle-même. Mais si on passe de ces principes d'analyse sociologiques à des travaux de nature historique, il est clair que l'investissement ne précède pas le modèle culturel qui viendrait luimême avant le mode de connaissance. Au contraire, c'est souvent la connaissance ou la moralité qui précède l'investissem $4 \sim$ nt dans 
un processus de changement historique. L'analyse de la structure sociale ne permet pas de répondre à de telles questions. Seule l'analyse du changement en est capable.

En revanche, c'est bien au niveau de cette définition de types généraux de sociétés et de niveaux d'historicité que se situe la notion de classe sociale car on ne peut pas séparer les rapports de classes du travail d'accumulation et par conséquent des autres dimensions de l'historicité. La classe dirigeante est celle qui prend en charge et s'approprie les moyens et les résultats de l'historicité, de la production de la société par elle-même. La distance de la classe dirigeante au reste de la population est celle qui sépare l'historicité de l'organisation sociale, la production de la société de son fonctionnement. C'est pourquoi les rapports de classes ne sont pas définis par la nature du groupe social qui dirige l'économie ou l'ensemble de la société mais par la nature de l'intervention de la société sur elle-même. Ce qui veut dire concrètement que les rapports de classes se situent au niveau d'un type de société et plus précisément de ce que je nomme un système d'action historique. Il existe un système de classes propres à la société industrielle et ce système est le même dans les sociétés dites capitalistes et dans celles qu'on nomme socialistes. Dans tous les cas, la classe dirigeante est formée de ceux qui ont la capacité d'utiliser le capital pour transformer l'organisation du travail et d'imposer ce mode d'organisation aux travailleurs. Chacun sait que cette domination de classe prend des formes historiques très différentes dans les différents régimes mais un mode de production ne peut pas être confondu avec ce que je nomme ici régime et que je nommerai plus tard, plus précisément, un mode de dév1eloppement. L'idée qu'on puisse définir un état de la division technique du travail, indépendamment d'une division sociale du travail qu'on nommerait système des rapports de classes, est dénuée de tout sens. La division technique du travail n'est qu'une forme appliquée de la division sociale du travail, c'est-à-dire des rapports de classes comme je l'ai indiqué dès le début en rappelant que la division technique du travail était produite par un système d'autorité qui était luimême la projection d'une domination de classe sur une organisation particulière. J'ajoute une fois de plus qu'on ne peut pas concevoir une société douée d'historicité ou pour parler plus vite histo- 
rique et en particulier une société capable d'accumulation qui ne soit pas une société de classes. Vouloir une société sans classes ne peut avoir d'autre sens que d'imaginer une société en deçà ou au delà de l'historicité. Dans une telle société, les rapports [162] de domination sociale peuvent en effet disparaître et leur place être occupée soit par une domination qu'on peut appeler biologique, celle des hommes sur les femmes, soit par la domination née de la conquête. On peut imaginer que nos sociétés historiques viennent de sociétés préhistoriques marquées surtout par la domination des hommes sur les femmes et qu'elles vont vers des sociétés post-historiques qui ne seront nullement des sociétés d'égalité mais celles où le règne de l'État aura remplacé complètement celui de la classe dirigeante. Si on s'écarte de ces visions très générales, il faut reconnaître que toutes les sociétés qui possèdent un système d'accumulation et d'investissement sont des sociétés de classes. La classe dominante autant que la classe dominée imagine une société sans classes - la classe dominante parce qu'elle veut s'identifier aux lois de la nature ou au mouvement de l'histoire et cacher ainsi ses intérêts particuliers ; la classe dominée parce qu'elle cherche à se ré approprier le contrôle des instruments et des résultats de l'historicité. L'idée de société sans classes est ainsi de la plus grande importance idéologique mais il est absurde d'opposer des sociétés de classes à des sociétés sans classes. Cette idée simple est importante dans le monde contemporain où l'habitude a été prise d'opposer les sociétés capitalistes aux sociétés socialistes comme si les unes étaient dirigées par une classe dirigeante tandis que les autres, quels que soient leurs qualités ou leurs défauts, seraient des sociétés sans classes. Les rapports de production sont fondamentalement les mêmes dans toutes les sociétés industrielles, quelle que soit la propriété des moyens de production.

Aucune notion n'est plus souvent employée en sociologie que celle de classe sociale et pourtant dans presque tous les cas son emploi repose sur un grave malentendu. Expliquer des conduites en les rapportant à la situation de classe de leur auteur aboutit en fait à réduire la notion de classe à celle de strate et par conséquent à placer cette notion de classe dans l'ordre de la consommation qui n'est pas le sien. Ceci n'enlève rien à l'intérêt des études sur la stratification sociale et sur l'inégalité mais il est possible de montrer que les enfants de familles aisées ou fortement éduquées ont plus de chance de parvenir à l'en- 
seignement supérieur que les enfants des ouvriers ou des agriculteurs sans avoir à recourir à la notion de classe sociale. On sait qu'en France Baudelot et Establet, sensibles à cette critique, se sont efforcés de montrer qu'il y avait non pas de l'inégalité devant l'école mais deux écoles, deux filières scolaires. L'idée est beaucoup plus intéressante : malheureusement elle est largement contredite par les faits, le plus important étant qu'un grand nombre d'enfants venant de catégories à statut socio-économique bas s'efforcent d'entrer dans l'école secondaire, y parviennent de manière non exceptionnelle mais sont beaucoup plus souvent refoulés. Cette fonction de refoulement qui pourrait nous guider vers une analyse en termes de classes sociales est très insuffisante dans l'œuvre de ces sociologues. Innombrables sont les travaux qui affirment péremptoirement qu'ils apportent une contribution à l'étude des classes en décrivant tel ou tel aspect de l'inégalité sociale. On ne voit pourtant pas comment l'idée de classe, pourrait être utilisée pour l'étude des différences dans les niveaux de consommation. Le véritable usage sociologique de la notion de classe sociale ne consiste pas à définir des catégories sociales ou des genres de vie mais à se demander dans quelles conditions les membres d'une classe agissent en tant que tels. En d'autres mots [163] le problème important est celui de l'action de classe. Il est patent que les conduites de classe ne sont pas constamment les plus visibles et les plus importantes. Les ouvriers de l'industrie, même lorsqu'ils se situent dans les lieux les plus favorables à la formation de la conscience de classe, n'agissent pas toujours à ce niveau-là. Ils agissent parfois comme groupes revendicatifs ; à l'inverse il est fréquent qu'ils agissent au nom de leur situation nationale plutôt qu'au nom de leur situation de classe. Tel est le paradoxe qui mérite d'être examiné : pourquoi la situation de classe qui apparaît comme un élément essentiel de l'analyse des sociétés modernes est-elle si loin de se traduire dans des conduites de classe qui domineraient nettement l'histoire ? Untel problème ne peut pas être traité indépendamment d'une réflexion plus générale sur les mouvements sociaux. L'important ici est de souligner que c'est dans ce domaine que la notion de classe trouve à s'employer, puisque c'est ici qu'une classe agit en fonction des rapports conflictuels où elle est placée et donc au niveau de la production de la société par elle-même et non d'une consommation préalablement coulée dans les catégories de la pratique sociale. L'autre remarque qui s'impose pour orienter correctement l'étude 
des classes sociales est qu'aucune classe n'agit uniquement de manière conflictuelle au nom d'intérêts économiques. La violence de conflits d'intérêts n'est pas suffisante pour prouver l'existence de conflits de classes. On a vu dans beaucoup de pays les petits commerçants réagir très vigoureusement contre la concentration du commerce et de la même manière, depuis beaucoup plus longtemps, des agriculteurs indépendants réagir avec force contre la pénétration des entreprises marchandes et industrielles à la campagne. Ir s'agit là de conflits d'intérêts très importants et qui ne peuvent pas être nommés des conflits de classes. Encore une fois il est impossible de définir une classe autrement qu'en termes d'action de classe. Une classe n'est pas une situation mais un acteur qui combat contre l'autre, contre la classe opposée pour le contrôle de l'historicité et au-delà pour accroître son influence institutionnelle et modifier à son profit le système d'autorité qui régit les organisations. Toute classe agit dans deux directions à la fois. Elle combat son adversaire et elle élabore un projet de gestion sociétale ; elle invoque ses intérêts et se réfère à des orientations culturelles qu'elle partage en tant que telles avec son adversaire social tout en les interprétant de manière opposée. Ainsi l'appel aux classes sociales comme élément central d'analyse de la société, loin de nous enfermer dans un objectivisme superficiel et qui se réduit vite à ce qu'il est en réalité, c'est-à-dire une forme inutilement compliquée de fonctionnalisme conduit à se représenter la société comme un système d'action. Le conflit des classes ne répond pas à une situation ; il la constitue ou plutôt le conflit des classes est le mouvement critique par lequel émerge à la surface de l'histoire l'opposition des acteurs de classe constamment cachée par les catégories de la consommation et celles de la reproduction sociale, en un mot par l'ordre établi. C'est ce qui donne son importance centrale à l'étude des mouvements sociaux, à travers lesquels s'opère cette remontée critique, cette révélation de la production de la société par ellemême qui est toujours indissolublement orientation culturelle et conflit social. Nous avons les plus grandes difficultés aujourd'hui encore à accepter une telle représentation de la société et par conséquent un tel rôle pour la sociologie, car nous sommes habitués depuis trop [164] longtemps à dissocier la structure et les acteurs comme nous l'ont recommandé tant de sociologues qui ne voyaient pas que par ce conseil ils détruisaient l'objet de notre 
travail. La société est un système d'action ; elle n'est ni simple interaction ni système définissable indépendamment des acteurs. Ceux-ci ne répondent pas à une logique non sociale, par exemple à la maximation des avantages ou à la minimisation des coûts; ils sont fondamentalement engagés dans leur travail de production conflictuelle de la société. Mais n'est-ce pas cette image qui correspond le mieux aux situations que nous avons à observer? N'est-il pas évident que l'idée de la logique d'un système économique a été remplacée par celle de systèmes de formation des politiques économiques? Lorsque nous discutons de l'avenir économique de nos sociétés ne sommes-nous pas obligés de parler directement en termes d'action sociale, de conflit et de négociation, en même temps d'ailleurs que d'innovation ou de défense des privilèges ? La conséquence la plus positive de l'idée que tout est politique est que nous devons nous débarrasser de l'idée que la politique est subordonnée à l'économie ou aux exigences du développement des forces de production. li est vrai qu'à chaque instant chacun de nous se sent et se sait placé devant une situation et qu'il décrit celle-ci en termes quantitatifs ou de stratification, mais le rôle de l'analyse sociologique est de s'écarter de cette pensée sociale spontanée, fausse du seul fait qu'elle part de la conscience individuelle et qu'elle est amenée ainsi à reconnaître la priorité de l'organisation collective de la société sur les conduites de chacun. Oui, tout est politique c'est-à-dire que toutes les formes d'organisation sociale et culturelle dès qu'on les examine d'un peu près, perdent leur caractère d'objectivité et apparaissent pour ce qu'elles sont, le résultat de relations politique de rapports de classes et d'orientations culturelles.

Une fois qu'on a présents à l'esprit ces principes élémentaires d'analyse sociologique, il est nécessaire d'entreprendre la reconstruction de tous les aspects de la vie sociale car une société ne peut pas être réduite à son champ d'historicité c'est-à-dire à l'affrontement des classes pour la direction de l'historicité. J'ai longuement étudié dans Production de la société et dans quelques autres textes le passage du champ d'historicité au système institutionnel puis aux organisations. Je n'y reviens pas ici. Mais il semble indispensable de donner en quelques mots une vue plus com- 
plète de la vie d'une société. Ce qui peut être fait de la manière suivante.

[165]

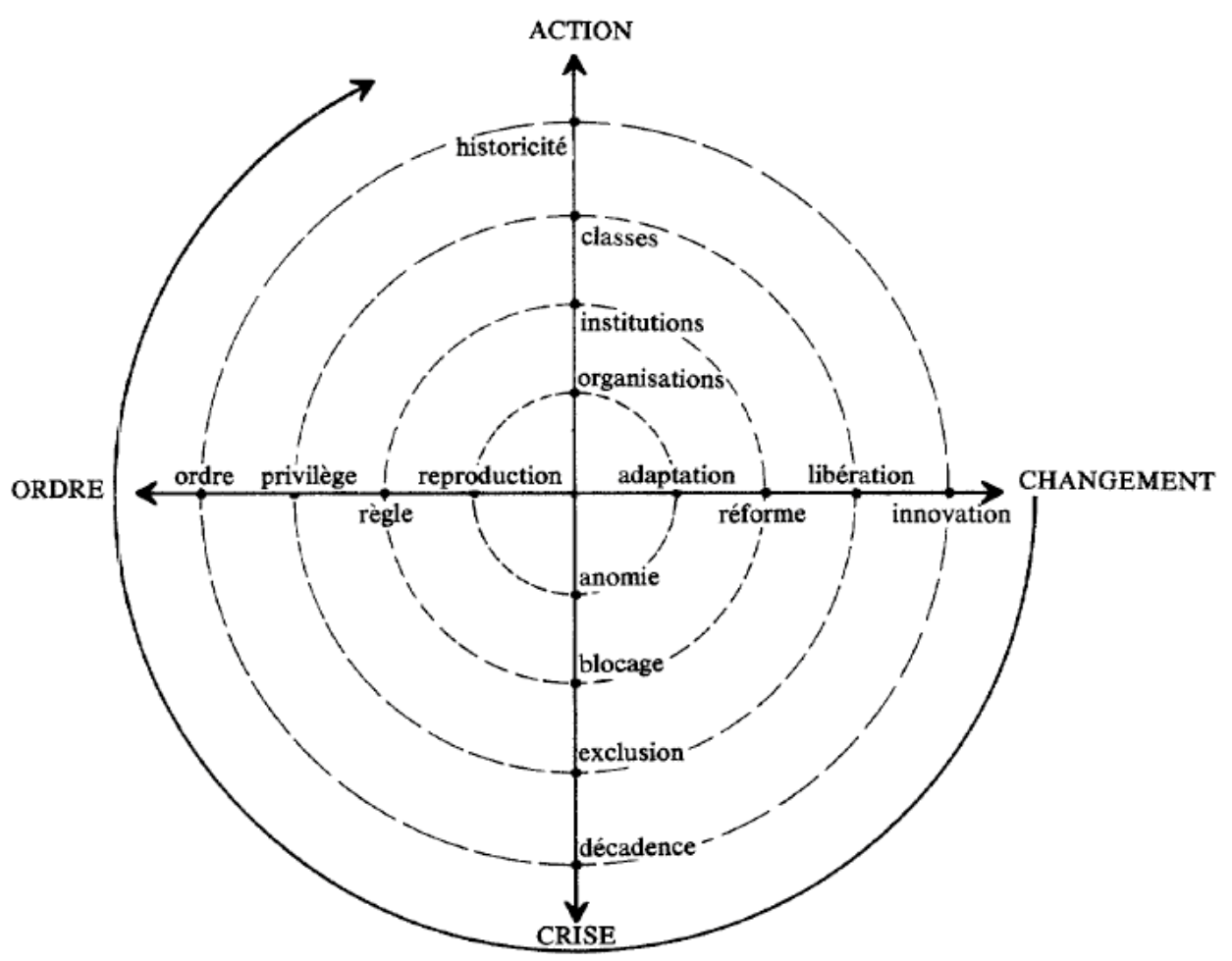

TABLEAU DE L'ANALYSE SYNCHRONIQUE

L'ensemble des systèmes d'action met en œuvre la production de la société par elle-même, mais cette œuvre ne s'accomplit qu'à l'intérieur d'une collectivité concrète, par exemple d'une société nationale, régionale ou locale, donc d'une unité territoriale. C'est dans ce cadre que les systèmes d'action et plus généralement la production de la société par elle-même se transforme en ce qui en est presque le contraire, à savoir des systèmes de reproduction. Il ne s'agit plus alors de l'action mais de l'ordre. La reproduction de l'ordre s'opère aux mêmes niveaux que la production de l'action sociale. Au niveau organisationnel de l'action correspond la socialisation, l'éducation qui placent les acteurs dans l'ordre social. L'équivalent du système institutionnel, système de relations poli- 
tiques, est l'ensemble des règles qui trace la frontière entre le permis et l'interdit, on peut parler ici de système juridique, en un sens élargi. La place occupée par les rapports de classes dans le domaine de l'action est occupée par la distance sociale et par conséquent par les privilèges dans celui de l'ordre. Celui-ci occupe en haut de son domaine la même place que l'historicité en haut du sien. Mais il ne suffit pas d'opposer ces deux axes. Les rapports sociaux dans la mesure même où ils sont soumis aux catégories de l'ordre, se décomposent, entrent en crise, de sorte que la hiérarchie des systèmes d'action a pour contrepartie une hiérarchie des formes de crise. L'anomie est la forme principale de la crise de l'organisation sociale. [166] Une société bloquée est une société dont le système institutionnel est en crise, soit qu'il soit trop rigide, soit qu'il soit décomposé. Des rapports de classes en crises sont en fait des rapports d'exclusion dans lesquels le conflit des acteurs est remplacé par l'opposition du in et du out. Enfin on peut nommer décadence la crise de l'historicité. À leur tour les catégories de la crise conduisent aux catégories du changement par lesquelles un ordre différent cherche à se constituer. L'ordre et le changement se placent donc sur un même axe, de même que l'historicité et la crise. Un changement organisationnel est une adaptation ; on nomme réforme une adaptation institutionnelle.

Le changement au niveau de rapports de classes en crise, changement qui combat les privilèges et la distance sociale, est une libération et le contraire de l'ordre, au niveau culturel, est l'innovation. Ainsi est constituée ce qu'on peut nommer une analyse générale de la société à condition de préciser qu'il ne s'agit en réalité que d'une analyse de la structure sociale et non pas des transformations historiques, bien qu'y figurent déjà des catégories comme ordre et changement. Ce tableau comporte deux enseignements principaux. Le premier est qu'il existe en sociologie des théories régionales, une théorie de l'ordre et de la reproduction, une théorie de la crise et une théorie du changement qui possèdent une large autonomie par rapport à l'étude de l'action et en particulier de l'action historique, c'est-à-dire de l'historicité. C'est pourquoi si souvent on parle d'écoles en sociologie, non pas seulement pour opposer des conceptions générales de la société mais pour opposer des théories régionales, dans la mesure où chacune 
de celles-ci tend à s'identifier à l'ensemble de la recherche sociologique, soit délibérément soit plus simplement parce qu'elles ne se préoccupent pas de se situer par rapport à d'autres modes d'analyse. La deuxième idée qu'impose cette figure est que l'autonomie des théories régionales a pour contrepartie la hiérarchie des domaines d'analyse. De la même manière qu'à l'intérieur de l'étude de l'action la connaissance des organisations est subordonnée à celle des institutions et celle-ci à son tour à la connaissance du champ d'historicité de même l'étude de la crise est toujours l'étude d'un ordre en crise et l'étude d'un ordre est toujours celui du renversement de la production en reproduction, de l'action historique en ordre établi. L'autonomie des théories régionales n'a d'autre limite que l'existence d'une théorie générale de la société qui consiste avant tout à définir précisément les formes d'emboîtement des théories régionales les unes dans les autres et la nécessité de remonter à partir de faits relevant du changement, de la crise ou de l'ordre jusqu'aux orientations culturelles et aux rapports sociaux fondamentaux C'est dire que tout le travail de la sociologie consiste à remonter à partir des changements, des adaptations, des réformes et des innovations, à travers les diverses formes de crise et par-dessus les appareils de l'ordre, vers les formes d'action qui se trouvent au sommet de la hiérarchie des systèmes d'action, vers les mouvements sociaux. L'étude des mouvements sociaux est le centre de la sociologie ou tout au moins le centre de l'étude des systèmes sociologiques. Affirmation paradoxale et qui se heurte à deux objections évidentes. Du côté de la classe dirigeante, ce qui se voit d'abord c'est la puissance de l'argent, des machines, du profit, comment parler de ce côté-là de mouvement social ? Et de l'autre côté, celui du peuple ou de la classe dirigée, et dominée, [167] il est vrai qu'on aperçoit des mouvements sociaux mais ils sont loin de remplir toute la scène historique. La vie des travailleurs industriels n'est pas une permanente lutte de classe. Elle est d'abord et avant tout fatigue, monotonie, attente du salaire, parfois aussi espoir de promotion ou de mobilité. Notre vie quotidienne n'est pas un drame ; elle est davantage une routine, le respect de règles, de codes, de hiérarchie. Ne sommes-nous pas pris dans la machine sociale et n'est-ce pas à des moments assez exceptionnels que nous pouvons avoir l'impression de la remettre en cause et ces moments particuliers Ile sont-ils pas davantage marqués par la violen- 
ce d'une crise historique, guerre ou catastrophe économique, que par l'action volontaire et organisée de mouvements sociaux ? Et pourtant rien ne peut dispenser de reconnaître la présence des mouvements sociaux au cœur de la vie sociale. Il faudrait le plus vite possible découvrir qu'une classe dirigeante est un acteur historique et non pas une loi économique ou la logique d'on ne sait quelle rationalité du profit. La différence avec la classe dominée est que la classe dirigeante n'oppose pas son idéologie à un ordre mais qu'elle donne à son idéologie la figure de l'ordre. Parce qu'elle est dominante, elle parle au nom de la totalité, de l'intérêt général ou du sens de l'histoire. Mais ce n'est pas pure fiction. Une classe dirigeante prend effectivement en charge l'historicité d'une société en même temps qu'elle la réduit à ses propres intérêts. Elle est dirigeante et dominante à la fois, elle est créatrice en même temps qu'exploiteuse. Il ne va pas de soi que les privilégiés ou plus simplement les riches se conduisent en classe dirigeante. Nous en avons une longue expérience en France où pendant de longues périodes de notre histoire moderne la bourgeoisie s'est davantage comportée comme un groupe de privilégiés que comme un agent d'innovation de sorte que plus d'une fois c'est l'État qui a dû assumer la charge principale de l'industrialisation.

La solidité de ma démarche ne me semblera établie que lorsque j'aurai été capable d'étudier le mouvement social d'une classe dirigeante par la même méthode que le mouvement social d'une classe dominante. Je dois cependant aujourd'hui m'en tenir à des observations sur les mouvements de ce dernier type. Observations brèves car j'ai si souvent traité de ces thèmes que le plus urgent est maintenant de passer à des études de cas et de mettre au point la méthode qui doit les permettre. Mais il est utile de réagir une fois encore contre ce qu'on pourrait nommer une conception théâtrale des mouvements sociaux. Comme si ceux-ci étaient des acteurs apparaissant sur la scène de l'histoire ou des personnages de roman doués d'intelligence et de volonté, nouveaux héros collectifs des grands drames de la vie sociale. Cette illusion est d'autant plus dangereuse qu'elle a été presque constamment entretenue par les partis politiques et plus encore par les États qui entendent parler au nom d'une classe ou d'une autre. Ils s'expriment souvent comme si la classe ouvrière avait une volonté en même temps qu'une organisation, en un mot comme si elle pouvait être entièrement identifiée à des dirigeants politiques qui seraient donc 
en toute circonstance habilités à parler en son nom. Le mouvement ouvrier, pas plus qu'aucun autre mouvement social, n'est un personnage. Il n'a ni tête ni membres ; il ne peut jamais être identifié à une organisation, syndicat ou parti même si la tradition historique a peu à peu fait apparaître l'image d'un mouvement ouvrier. Le sociologue ou l'historien savent [168] bien que syndicats, partis, mutuelles, coopératives, municipalités, associations culturelles, doctrines sociales forment autant d'éléments d'un ensemble qui reste toujours démembré et que seule l'analyse ne peut reconstituer. Il faut donc insister sur la complexité de tout mouvement social. Certes il faut dire d'abord qu'il est toujours constitué par le conflit avec un adversaire, la visée de l'enjeu de ce conflit et la lutte contre l'emprise de l'adversaire sur l'enjeu, ce que j'ai figuré par le triangle identité (1), opposition (0), totalité ( $\mathrm{T}$ ) et par les trois dimensions constitutives du mouvement : le conflit 10, le projet IT et le rejet de OT. Mais l'essentiel pour l'analyse est d'aller au-delà de cette formulation simple et de reconnaître au moins trois éléments de complexité. Le premier et le plus évident est qu'un mouvement social peut difficilement n'exister qu'à son propre niveau. Pour être fort, il faut qu'il incorpore et dépasse des revendications organisationnelles et des pressions institutionnelles. Pas de mouvement ouvrier sans revendication de salaire et sans pression pour obtenir en faveur du syndicat le droit de négocier. Inversement, pas de mouvement social si les revendications et les pressions ne sont pas triées en fonction de l'objectif souverain de la lutte de classe et du projet culturel. Il n'existe pas de solution parfaite et stable, pas de mouvement social qui parfois ne s'enfonce pas dans la simple revendication et parfois ne se perde pas dans des débats idéologiques qui perdent le contact avec la vie pratique. Un deuxième élément de complexité tient à ce que tout mouvement social a nécessairement deux faces : il combat une classe dirigeante mais il lutte aussi contre l'ordre établi, opposition symbolisée par celle des dimensions IO et OT mais qui renvoie plus largement au fait que les rapports de classes sont à la fois des rapports de production et des rapports de reproduction. Plus l'emportent les premiers, plus est important le mouvement social proprement dit; plus contraignants sont les seconds et plus devient importante l'action de rupture que j'ai nommée action critique. Sans action critique le mouvement social se réduit à une pression institutionnelle 
ou à une revendication organisationnelle ; sans institutionnalisation, le mouvement social n'est plus qu'action critique, c'est-à-dire en fin de compte participation à une œuvre de transformation de l'État et de mutation historique mais non plus action de lutte sociale proprement dite. Ici encore l'équilibre est toujours instable entre ces deux dimensions. Nous le savons particulièrement bien en France ou en Italie, pays où aujourd'hui encore le mouvement ouvrier est marqué à la fois par un effort d'institutionnalisation des conflits et par une volonté de lutte directe contre l'État. Le troisième élément de complexité que je veux mentionner est que tout mouvement social reste, même quand il est de niveau très élevé, imparfaitement intégré. Il ne se contrôle pas entièrement luimême et sa force historique vient en partie de ce que chacun des éléments qui le composent garde sa vie propre. La dimension du conflit, l'opposition à l'adversaire, garde toujours une certaine autonomie ; c'est pourquoi tout mouvement social a aussi des aspects de guerre civile et plus simplement de violence. La défense de l'acteur n'est pas seulement défense d'un rôle social ou d'une classe ; elle est aussi libération, affirmation de l'existence indépendante de l'acteur; pour le mouvement ouvrier elle est aussi refus du travail, absentéisme et même culture populaire, joyeuse ou brutale, mouvement de retrait et de rejet de la situation sociale. Enfin l'enjeu, l'historicité, si l'acteur veut le reconquérir, il veut aussi [169] s'en débarrasser, puisque cet enjeu reste toujours plus ou moins aux mains de l'adversaire. Dans tout mouvement social existe aussi comme l'a souligné Vidal une volonté de destruction, l'attirance de la mort et le souffle prophétique qui annonce la fin du monde.

Cette complexité des mouvements sociaux ne cesse de croître à mesure que l'aire de ces mouvements s'étend davantage, de sorte que plus une société est moderne et moins ces mouvements sociaux ont de chances de s'incarner dans des personnages ou des organisations, alors même que ces sociétés sont plus complètement agitées par les mouvements sociaux que toutes celles qui les ont précédées. On peut se demander si le mouvement ouvrier n'a pas été la dernière forme de mouvement social qui correspond à un personnage historique, à une classe sociale réelle. À partir du moment où les rapports de classes n'opposent plus des classes réelles, des groupes et des collectivités possédant une existence, une culture et une vie sociale autonome, on 
ne voit plus ce qui peut assurer l'unité historique d'un mouvement social. Il ne peut plus être qu'une idée, que le point vers lequel converge, par des chemins sinueux, une multiplicité de luttes conscientes de leur parenté mais incapables de s'intégrer entièrement les unes aux autres. Cette dernière remarque nous introduit déjà à une réflexion sur la société contemporaine. Il est donc temps de quitter les principes d'analyse des systèmes sociaux et d'aborder la deuxième moitié du travail de sociologue, l'étude du changement social.

\section{L'ANALYSE DIACHRONIQUE}

$\underline{\text { Retour à la table des matières }}$

La pensée sociale dont nous avons hérité pouvait difficilement concevoir une analyse du changement social. Non qu'elle fut préoccupée seulement de ce qui était stable, mais au contraire parce qu'elle reposait sur l'idée d'évolution. Tant que la sociologie reste évolutionniste, elle ne peut pas concevoir le changement social car elle ne peut pas dissocier l'analyse du système social et celle du changement. L'analyse synchronique occupe une place plus centrale que l'analyse diachronique, non pas pour des raisons de principe, mais parce que c'est elle qui a rompu avec l'évolutionnisme et qui a ainsi rendu possible une théorie du changement. C'est très récemment que cette idée a été acceptée, au moins dans les pays où la pensée historique avait connu ses principaux succès, l'Allemagne, la France, l'Angleterre et l'Italie. Pendant longtemps se fit entendre dans ces pays une opposition quasi-nationale aux thèmes de la sociologie fonctionnaliste qui semblait alors identifiée à la pensée américaine. Faux combat s'il en était, avant tout parce que Parsons indiqua très clairement que son analyse de la société reposait sur une conception évolutionniste, sur l'idée, héritée du siècle passé, que le mouvement de l'histoire conduit vers plus de rationalité instrumentale. Je n'ai pas à revenir ici sur les causes du déclin de l'évolutionnisme. Il est plus important d'insister sur les dangers de ce qui semble avoir pris la place de l'évolutionnisme et qu'on peut nommer l'historicisme. La différence entre les deux est que l'évolutionnisme, d'inspiration plus anglaise, pense dégager des tendances générales de l'évolution sociale, à mesure que les sociétés deviennent plus techniques et plus complexes, tandis que l'histori- 
cisme au contraire, d'inspiration allemande, insiste sur la particularité du parcours de [170] chaque acteur collectif, guidé par une volonté et orienté par une culture et par une histoire. Aujourd'hui où l'histoire est occupée surtout par la multiplicité conflictuelle des modèles de développement, l'historicisme triomphe, tandis que l'évolutionnisme régresse. Les sociétés qui croyaient parler au nom de valeurs universelles se sont vu rappeler, souvent brutalement, qu'elles avaient été des sociétés colonisatrices, qu'elles étaient encore les centres de l'impérialisme et qu'elles faisaient peser leur puissance économique et militaire sur une grande partie du monde. La crise culturelle des années soixante a également mis fin à l'illusion d'une évolution linéaire conduisant vers plus d'instrumentalité, de séparation des rôles et de froideur dans la vie sociale. Le danger de l'historicisme est d'enfermer chaque société dans sa particularité, c'est-à-dire de faire disparaître les sociétés derrière les États, les systèmes sociaux derrière les politiques, et plus simplement les pratiques derrière les discours. C'est pourquoi la tâche la plus importante et la plus pratique de la sociologie aujourd'hui est de définir les relations entre l'analyse des systèmes sociaux et l'analyse des transformations historiques, entre l'analyse synchronique et l'analyse diachronique. Ce qui suppose d'abord qu'on reconnaisse leur séparation. La manière la plus simple de l'affirmer est de reconnaître qu'il n'existe jamais de changement historique, de passage d'un type de société à un autre, d'un champ d'historicité à un autre, qui soit purement endogène. Tout changement social est à un degré ou à un autre exogène. Ce qui rend désuète l'idée de la deuxième Internationale selon laquelle un type de société ne pouvait se développer que quand le type précédent avait épuisé toutes ses virtualités. Même les sociétés les plus dominantes ne se transforment pas par la simple accumulation de leurs techniques, de leurs richesses et de leurs échanges. Elles sont aussi exposées que les sociétés dépendantes ou colonisées à des causes extérieures de changement et plus précisément à des facteurs non sociaux, à des facteurs de concurrence économique et militaire. La guerre est de plus en plus un facteur important du changement sociétal. Dans le passé la conquête guerrière a souvent superposé un ordre étatique à une vie économique locale qui n'en était pas fondamentalement transformée. Dans d'autres cas la conquête a placé une économie marchande ou industrielle au des- 
sus d'une économie agraire, mais aujourd'hui les liens de la recherche scientifique et technologique, des grands investissements économiques et des stratégies militaires sont tellement étroits qu'il est impossible de parler du passage interne d'une économie industrielle à une économie post-industrielle. Si la création de techniques modernes relève encore en Union Soviétique du domaine réservé de l'État et de l'armée, il n'en va pas de même aux ÉtatsUnis et dans les grands pays occidentaux où les choix militaires et stratégiques ont des effets beaucoup plus étendus sur les investissements et l'organisation générale de la production. Plus les sociétés sont «modernes » et plus aussi elles sont fragiles, plus elles dépendent de modifications survenues dans leur environnement. Cette vieille idée, au nom de laquelle on explique depuis longtemps les progrès économiques plus rapides des sociétés maritimes est plus utile aujourd'hui que jamais. On peut concevoir des sociétés refermées sur elles-mêmes, réduisant autant que possible la pression de l'environnement économique et politique. De telles sociétés peuvent transformer profondément leur organisation sociale, comme ce fut le cas pour la Chine et plus encore pour le Cambodge, mais elles ne sont pas entraînées vers une transformation de [171] leur capacité de production. C'est au contraire le risque militaire d'un côté et la volonté de jouer un rôle international de l'autre qui poussent aujourd'hui la Chine à développer des formes modernes de production. De la même manière au $\mathrm{XIX}^{\mathrm{e}}$ siècle, c'est la menace que faisaient peser sur le Japon les flottes américaine et russe qui déclencha la révolution Meiji et l'industrialisation accélérée du pays. Une analyse de la société comme ensemble de système d'action a pour contrepartie nécessaire la reconnaissance du caractère exogène du changement. Ce qui doit conduire à une idée plus générale. Les acteurs principaux du chang1ement sociétal ne peuvent pas être les mêmes que ceux qui sont au centre du fonctionnement d'une société. D'abord il n'est pas possible de parler de transformation de la société capitaliste alors qu'on peut parler de son fonctionnement. La société industrielle ou capitaliste est un système social ; ce qui change est l'Angleterre ou le Japon, c'est-à-dire une société politique, territoriale, définie historiquement et géographiquement. L'identité de cette société nationale est représentée non par une classe dirigeante mais par un État. L'État est l'agent qui représente une société dans ses rapports intersociaux; il est du même coup l'expression d'une société comme 
acteur de sa propre histoire. Il établit la relation du présent avec le passé et l'avenir comme la relation de l'intérieur avec l'extérieur. Il n'y a pas d'État qui n'ait pas le droit de paix et de guerre. Il n'yen a par conséquent pas qui n'ait pas la capacité d'engager la vie et l'avenir d'une société. Mais il n'yen a pas non plus qui ne soit pas le garant de 1'0rdœ social, c'est-à-dire de l'ensemble des mécanismes de reproduction. L'État se situe sur l'axe de l'ordre et du changement et non pas sur celui de l'action et de la crise. Ce qui semble plus facile à accepter si on distingue clairement l'État du système politique. Ici encore il faut en finir avec l'ethnocentrisme propre aux sociétés capitalistes centrales dans lesquelles l'État, au moins à l'intérieur des frontières, semble souvent se confondre avec le gouvernement, voire même avec les représentants élus du peuple. Le système politique est un système de représentation des intérêts sociaux ; il est par conséquent subordonné aux rapports de classes tout en possédant une autonomie qui tient d'abord à la complexité de toute société nationale, de toute formation sociale. Dans les sociétés libérales, cette autonomie est grande et l'importance du système politique : est si considérable que l'État semble presque disparaître. C'est bien dans l'Angleterre où vivait Marx que l'État a le plus dépéri mais on ne peut oublier, même dans ces cas-là que cet État était bien vivant dès lors qu'il s'agissait de développer et de maintenir l'empire et de faire face à la concurrence des autres nations industrielles. Rien n'est plus superficiel et même faux que d'affirmer l'identité de l'État et de la classe dirigeante. Que la classe dirigeante à travers la domination qu'elle exerce plus ou moins sur le système politique ait une grandie influence sur l'État, nul ne l'a jamais oublié mais le phénomène historique le : plus important et le plus constant est la distance qui sépare l'État de la classe dirigeante. Le développement économique de l'Italie, de l'Allemagne et du Japon fut rendu possible par des initiatives étatiques beaucoup plus que par l'action des bourgeoisies nationales et dès qu'on s'éloigne un peu plus des centres de l'industrialisation capitaliste, le rôle de l'État dans les transformations historiques est si évidemment dominant qu'il semble absurde d'en faire le serviteur d'une classe dirigeante qui en général n'existe même pas ou que cet État s'empresse de liquider. En Algérie comme au Brésil, au Mexique comme [172] en Iran, au Viet-Nam comme au Congo, en Irak comme en Pologne, c'est bien l'État qui dirige l'industrialisation et la transformation de la société. Il est vain de chercher à réduire ce pouvoir d'État à une réalité de classe 
en parlant par exemple de bourgeoisie d'État. Les deux mots n'appartiennent pas au même vocabulaire et leur accouplement ne fait que masquer un problème qu'il est indispensable de traiter directement. Assurément l'État n'est pas étranger aux intérêts et aux rapports de force qui dominent la société civile mais cette constatation banale ne justifie aucunement qu'on confonde deux ordres de problèmes, ceux du fonctionnement et ceux de la transformation des sociétés. Mais cette distinction ne devient claire que si on n'oppose pas seulement l'État à la société civile. Ce qu'il faut distinguer c'est plus largement le mode de production et le mode de développement d'une société. Ce qui conduit à remettre en cause les idées les plus communément admises. J'ai déjà dit que les classes et les rapports de classes se situaient dans un mode de production ou plus précisément dans un type d'historicité et en particulier d'investissement. Il existe des rapports de classes propres à la société industrielle et j'ai souligné à ce propos que ces rapports de classes étaient les mêmes dans une société capitaliste et dans une société socialiste. Ce qui conduit à dire que ce qu'on nomme capitalisme et socialisme ne sont ni des modes de production ni des rapports de classes mais des modes d'industrialisation. Le capitalisme est la création d'une économie marchande, industrielle ou post-industrielle par une bourgeoisie nationale ; on peut parler de capitalisme dépendant lorsque la transformation économique est dirigée par une bourgeoisie étrangère ou plus exactement ou par un système capitaliste dont le centre est à l'extérieur des pays considérés. On a souvent appelés socialistes des pays dont la transformation s'était opérée sous la direction d'un État national et en particulier en rupture avec la participation dépendante au système capitaliste mondial. Je souligne cette affirmation : le capitalisme n'est pas un mode de production, et j'ajoute même : le capitalisme ne définit pas des rapports de classes. Il est un régime, un mode de développement, une forme sociale de développement économique et en particulier d'industrialisation. La propriété des moyens de production est une chose, les rapports sociaux de production en sont une autre. L'accumulation capitaliste et l'accumulation socialiste, c'est-à-dire étatique, ont des formes extrêmement différentes mais leur signification de classe est la même dans la mesure où dans les deux cas elles installent des sociétés industrielles c'est-à-dire des ensembles de production où les travailleurs 
sont subordonnés à une organisation dite rationalisée du travail, dirigée par les détenteurs du capital. La séparation de ces deux domaines condamne le discours idéologique qui se réfère à une société nationale en la définissant en toute occasion comme socialiste ou capitaliste. Que des ouvriers à la chaîne soient soumis à des cadences de production épuisantes et à des systèmes de rémunération ou rendement n'a rien à voir avec le capitalisme mais est un des problèmes les plus importants des sociétés industrielles. D'une autre manière, on n'a pas le droit de dénoncer certains inconvénients, certains scandales des sociétés fortement industrialisées et urbanisées en en accusant le capitalisme ou inversement le socialisme. Aujourd'hui, quand on parle dans une société capitaliste industrialisée de passage au socialisme, que veut dire cette expression? Elle a plusieurs sens. Le plus concret est celui qui énonce la nécessité de renforcer l'intervention de [173] l'État dans l'économie, de développer le secteur public au dépens du secteur privé parce que celui-ci est incapable de faire face aux profondes transformations et aux investissements qu'exige un environnement international menaçant ou d'importantes innovations technologiques. Un deuxième sens, qui n'a aucune relation avec le premier, insiste sur la nécessité de renforcer l'influence ou les pouvoirs des travailleurs par rapport aux employeurs, privés ou publics, dans le travail et dans l'ensemble de la vie sociale. Quant aux expressions comme : transition vers le socialisme, qui laissent entendre que le socialisme est une étape de l'histoire postérieure au capitalisme, elles sont simplement dénuées de sens et il faut une fois de plus rappeler que si beaucoup de sociétés capitalistes industrielles sont devenues dans une certaine mesure socialistes, dans la mesure au moins où l'intervention de l'État s'y est fortement accrue, aucune société capitaliste n'est devenue socialiste au sens où on dit que l'Union Soviétique ou que la Chine sont socialistes. La vie politique, dans un pays comme la France, fait le plus grand usage d'expressions tout à fait vides de sens et que ceux qui les emploient à profusion se gardent bien de définir. On entend dire que tel parti n'entend pas faire passer le pays au socialisme mais se contenterait volontiers d'installer une démocratie avancée. Mais cette avance est à son tour conçue comme un premier pas vers le socialisme, c'est-à-dire vers l'étatisation des moyens de production. On ne satisfait pas ceux qui emploient de telles expressions si on leur disait 
qu'elles sont tout à fait acceptables à condition de préciser qu'elles n'impliquent aucune transformation de la condition des travailleurs et aucune modification des rapports de classes. Il est donc plus simple de renoncer à de tels slogans qui ne pouvaient avoir de sens qu'à l'époque où s'imposait naturellement une conception évolutionniste de l'histoire, comme si les régimes sociaux se succédaient dans la majestueuse caravane de l'histoire.

On ne peut s'en tenir là et se contenter de séparer l'étude du système et celle du changement. Chacun sent qu'elles ne sont pas entièrement isolables l'une de l'autre et, si elles se croisent, il faut que nous apprenions où situer leur point de croisement qui se trouve être le lieu central de l'analyse sociologique. Cette dernière expression doit être prise au pied de la lettre. Le problème central de l'analyse sociologique est bien celui des relations entre la synchronie et la diachronie, entre la classe dirigeante et l'État. De très nombreux penseurs sociaux ont eu depuis longtemps une très vive conscience de la place centrale qu'occupe cette interrogation dans toute conception de la société. Aux formulations déjà employées s'en ajoute une autre : quelle est la relation entre les mouvements de classe et les mouvements nationaux car les mouvements de classe occupent une place centrale dans le fonctionnement de la société tandis que les mouvements nationaux sont les actions collectives les plus importantes dans l'ordre du changement historique, puisque celui-ci est dominé toujours par la figure de l'État. Question mieux connue des historiens que des sociologues, qui est apparue pour la première fois de manière éclatante dans la pensée austro-marxiste et n'a cessé de prendre de l'importance à mesure que des révolutions ou des transformations dites socialistes pénétraient plus largement dans des régions dominées par des États nationaux autocratiques ou des États étrang1ers colonisateurs.

L'analyse synchronique et l'analyse diachronique ne se croisent pas simplement; leur relation est inscrite dans l'analyse du système social et plus précisément dans celle des rapports de classe. Car ceux-ci, on le sait, ont deux faces : rapports de production entre une classe dirigeante et une classe travailleuse et revendicative ; rapports de reproduction entre une classe dominante et une classe dominée. D'un côté les classes antagonistes 
luttent pour le contrôle de l'historicité et plus leur conflit est vif et plus aussi il développe les forces de production et de changement par rapport aux facteurs de crise et aux forces de reproduction. $\mathrm{Au}$ contraire, lorsqu'une classe dominante défend surtout ses privilèges et qu'une classe populaire défend son genre de vie traditionnel, elles n'ont besoin ni l'une ni l'autre de se référer à l'historicité et cherchent alors l'État comme champ de leur conflit ou comme allié contre adversaire. C'est la distance entre les rapports de production et les rapports de reproduction qui détermine la distance entre la classe dirigeante et l'État. Partout où, pour les raisons les plus diverses, la classe dirigeante est en réalité une classe dominante, celle-ci doit s'appuyer sur l'État pour faire respecter l'ordre qui la favorise et réprimer les tentatives pour le transformer. Les classes populaires de leur côté doivent alors s'attaquer à l'État protecteur principal d'une classe dominante qui sans l'appui de ses armes et de ses lois serait incapable de se défendre. Là où l'État n'est que l'agent corrompu ou artificiellement maintenu d'un État colonisateur, la lutte sociale peut même cesser presque complètement d'être sociale et se concentrer sur le terrain de la lutte armée contre l'État. Tel fut le cas de la guérilla cubaine contre Batista, tel est encore le cas de la guérilla menée au Nicaragua et si les guérillas échouèrent au Vénézuela, au Pérou ou même en Bolivie, c'est parce que l'État, dans ces pays, était loin d'être semblable à celui qui renversa Fidel Castro ou à celui des Somoza. En France c'est parce que l'industrialisation a été menée plus souvent par l'État que par la bourgeoisie et parce que celle-ci a été fort occupée à défendre ses privilèges et à constituer un bloc des intérêts oligarchiques que l'action du mouvement ouvrier a été souvent subordonnée à l'entreprise communiste menée contre l'État. En revanche dans l'Angleterre de la fin du XIX ${ }^{\mathrm{e}}$ et du début du XXe siècle ou aux États-Unis depuis la création de l'AF of L, l'action ouvrière a été presque complètement dissociée d'une action contre l'État et en revanche fortement associée à l'action de partis politiques visant à des transformations institutionnelles. Plus on s'approche du centre de l'économie capitaliste et plus la société civile semble dominer l'État, au point que celui-ci peut n'apparaître que comme l'agent de la classe dirigeante. Plus on s'éloigne de ces pays centraux et plus on rencontre des situations différentes mais dont les effets sont les mêmes au niveau général 
d'analyse que nous considérons ici. D'un côté les pays dépendants ou colonisés étant subordonnés à une initiative économique externe sont largement dominés par des classes dirigeantes, des oligarchies. De l'autre côté les pays qui n'ont pas été colonisés mais qui n'ont pas été emportés dans le premier mouvement de l'industrialisation sont ceux où l'État intervient comme défenseur d'un ordre social au service d'anciennes classes dominantes qui n'exercent plus de rôle économique dirigeant et innovateur. Dans tous ces cas l'action politique l'emporte sur la lutte sociale. Ainsi c'est dans l'analyse synchronique elle-même que se trouve l'explication de ses rapports avec l'analyse diachronique, ce qui lui confère un privilège décisif dans l'analyse. Mais, on vient de le [175] voir, c'est aussi largement dans la domination exercée par un État, qu'il soit autocratique et conservateur ou qu'il soit conquérant au service d'un capitalisme dynamique, que réside l'explication de la disjonction de la classe dirigeante et de la classe dominante et de la supériorité de celleci. Rien ne marque plus clairement la liaison entre les deux grands ordres d'analyses que la notion de révolution. Celle-ci associe par définition un mouvement social populaire ou de classe non dirigeante à une action politique de renversement de l'État. Ces deux éléments ne peuvent être réduits l'un à l'autre. Les paysans ou les artisans se soulèvent pendant la Révolution Française contre les propriétaires fonciers et contre les marchands ; en même temps la bourgeoisie renverse les privilèges et le pouvoir royal. L'histoire de la Révolution française est faite des relations changeantes entre ce mouvement social et cette action politique, Robespierre s'étant placé au point central de la Révolution du fait qu'il voulait combiner ces deux significations ; il fut à la fois l'homme de la terreur et celui du maximum. Mais sa chute témoigna de la fragilité de ce compromis et fut suivie du triomphe de la bourgeoisie. Le personnage de Lénine est plus important encore : parce qu'il a poussé plus loin cette même ambigüité. S'il fut surtout au plus profond de lui-même homme de parti et de lutte contre l'État ou de création d'un nouvel État, il fut aussi, en particulier au moment décisif où il écrivit l'État et la Révolution et les Thèses d'avril, l'homme du mouvement ouvrier et populaire et de la révolution sociale. Au cours de ses dernières années de gouvernement, s'il donne presque toujours le privilège à la construction de l'État et du parti, on sent souvent vivre en lui la volonté de rester l'interprète d'un mouvement social. Après sa mort s'accentuera la tendance qu'il avait déjà encoura- 
gée par ses actes les plus importants et le parti-État dévorera le mouvement social avec lequel il avait fait la révolution. On appelle stalinisme cet État qui dévore son père, mais ce même phénomène central se trouve dans presque tous les régimes communistes. C'est ce qui oppose le totalitarisme communiste au totalitarisme fasciste qui détruit ses adversaires sociaux plutôt que les forces qui l'ont mené au pouvoir.

Je ne reviendrai pas ici sur les transformations sociétales qui affectent aujourd'hui les sociétés industrialisées occidentales. Il n'est pas possible d'entrer ici dans une analyse suffisante de la société programmée ou post-industrielle.

Je rappelle seulement, en réponse à Louis Maheu, que la société programmée ne remplace pas plus la société capitaliste qu'elle n'en est un nouvel avatar. Il y a des sociétés programmées capitalistes et d'autres qui ne le sont pas comme il y a des sociétés capitalistes qui sont industrielles et d'autres qui sont soit marchandes soit programmées. Une fois de plus il faut se débarrasser de la tentation de définir une société nationale de manière unidimensionnelle.

En revanche il est nécessaire d'aborder l'analyse des nouveaux mouvements sociaux propres à cette société, parce qu'il s'agit là d'un thème de la plus grande urgence et de la plus grande difficulté. Il est en effet difficile de séparer dans les conduites collectives et les formes de contestation qui occupent de plus en plus souvent notre scène historique les luttes qui portent en elles ces nouveaux mouvements sociaux, celles qui manifestent la crise de la société industrielle et celles qui répondent à des difficultés conjoncturelles ou catégorielles. Au cours des dernières années l'idée s'est répandue, et elle a été reprise [176] ici par Alberto Melucci, que les conflits sociaux de la société industrielle étaient de plus en plus remplacés par des conflits culturels. La crise de la famille et de tous les modes d'éducation, le mouvement féministe, les campagnes menées par les homosexuels, pour ne prendre que quelques exemples d'importance très différente, ne montrent-ils pas que c'est aujourd'hui dans l'ordre du genre de vie, ce qui est plus large que la consommation, que se situent les conflits principaux qui se trouvaient dans la production à l'époque industrielle et probablement dans un cadre urbain ou territorial à l'époque marchande ? Plus profondément, le propre de la plupart de ces luttes n'est-il pas de remettre en cause le thème 
de la croissance et de chercher à établir de nouveaux équilibres ? Serge Moscovici a été plus loin encore lorsqu'il a affirmé que la question sociale était remplacée aujourd'hui par la question naturelle, le problème des rapports des hommes entre eux et des classes entre elle par celui des rapports de la société et de son environnement, par celui de la sauvegarde de l'écosystème dont nous faisons partie et que nous nous acharnons à détruire au péril de notre propre vie. Il faut prendre position sur des thèmes aujourd'hui aussi répandus, même si nous n'avons pas encore achevé l'ensemble d'études qui doit apporter une réponse précise à ces interrogations et à ces débats. Je prends nettement position contre l'idée de la substitution de conflits et de problèmes culturels à des conflits et à des problèmes sociaux; je m'oppose plus nettement encore à l'idée que nous entrons dans une société qui se trouve au delà du conflit des classes et qui doit surtout gérer ses relations avec les autres éléments de la nature. Les phénomènes de contreculture et de contestation culturelle sont un des éléments les plus importants de la crise de passage d'un type de société à un autre ; ils ne sont nullement caractéristiques de la société programmée. Celle-ci est, plus encore que la société industrielle, qu'elle prolonge en même temps qu'elle la dépasse, une société de production et d'accumulation. Dans cette société programmée, les conflits sociaux de classes sont plus généraux que dans les sociétés industrielles puisque des appareils de gestion et de domination sociale s'installent dans presque tous les domaines de la vie sociale. Surtout, ces conflits se transforment. fis ne se situent plus à l'intérieur des entreprises pour opposer les travailleurs aux dirigeants. Ils opposent les appareils aux populations dont ils ont la capacité de définir les besoins dans un domaine particulier ou en général. Il s'agit bien d'un conflit social mais qui se place en effet de plus en plus souvent dans l'ordre de la consommation, si on prend ce mot au sens le plus large. La technocratie nucléaire impose un mode d'organisation sociale ; les mouvements écologistes et antinucléaires, de manière utopique ou non, opposent à ce projet technocratique l'image d'autres types de sociétés, de production et de consommation, et combattent le pouvoir de la nouvelle classe dirigeante. Ce ne sont plus les travailleurs salariés et sans autorité de ces grandes organisations qui constituent la base sociale principale de ces nouveaux mouvements sociaux; mais, si nous savons qui 
sont les initiateurs de ces nouveaux mouvements, nous savons encore mal définir leur base sociale. C'est en termes d'usagers, d'habitants, voire d'êtres vivants, que cette base peut être le moins mal définie. Ce n'est en tout cas plus en terme de classe de travailleurs mais ces usagers sont bien une classe dans la mesure même où ils sont soumis au pouvoir d'une nouvelle classe dirigeante. Les luttes actuelles n'auront d'importance durable que dans la mesure où elles s'écarteront d'un rejet global du [177] monde de la production et de l'investissement et où elles sauront désigner leur adversaire au cœur du système de production et de consommation, adversaire capable aujourd'hui de commander la demande autant que l'offre. Une des tâches principales des sociologues d'aujourd'hui est de suivre, d'annoncer et de prévoir la naissance de ces nouveaux mouvements sociaux à travers les diverses formes et étapes de la crise et du déclin de la société industrielle. Il faut suivre la décadence du mouvement ouvrier, la crise culturelle plus large qui remet en cause les fondements de la société passée, parvenir au refus central de la croissance et à la recherche de nouveaux équilibres avant de rencontrer une critique libérale ou libertaire de l'État qui annonce et masque à la fois une nouvelle lutte sociale, s'arrêter un instant sur le repli en direction de groupes primaires ou de l'expérience vécue avant de découvrir, mêlés d'abord à la volonté de catégories menacées s'efforçant de retrouver leur identité tout en acceptant le changement, les nouveaux mouvements sociaux qui commencent à apparaître et dont jusqu'ici le mouvement antinucléaire représente la manifestation la plus importante.

Au moment où, dans les pays où le marxisme est la forme de pensée sociale la plus influente dans les départements de science sociale cette école de pensée recule brusquement, comment ne pas définir notre propre pensée dans le débat idéologique qui se trouve ainsi brutalement ouvert ? J'en ai assez dit pour que la distance entre la sociologie de l'action que j'expose et le marxisme soit évidente. Le marxisme est d'abord une recherche des lois objectives de l'histoire et son analyse n'est jamais menée en termes d'action sociale et de mouvements sociaux. Alors que je place au centre de ma réflexion le conflit, le marxisme organise la sienne autour de l'idée de contradiction, et, qui plus est, de : contradiction entre les comportements sociaux et des exigences naturelles, entre les rapports sociaux et les forces de production. À cette opposition fondamentale s'en sont ajoutées d'autres, 
plus conjoncturelles. La pensée marxiste, considérée comme celle de tous ceux qui se réclament du marxisme, est la doctrine qui a interprété l'idéologie du mouvement ouvrier. Lorsque celui-ci était le mouvement social principal, la pensée marxiste était vigoureuse, indépendante, critique et elle avait à mener de dures batailles contre les idéologies de la bourgeoisie pour se faire reconnaître. Ensuite la pensée marxiste a cessé d'être l'expression autonome d'un mouvement social pour devenir surtout la doctrine des partis communistes et dans une mesure beaucoup plus limitée de partis socialiste mais ceux-ci étaient à vrai dire après la première guerre mondiale et surtout après la seconde peu soucieux d'élaboration doctrinale. Cette association de la pensée marxiste et des partis communistes à l'échelle du monde et même de l'Europe occidentale a eu pour conséquence un dépérissement presque complet de la réflexion marxiste. Plus récemment encore, et à mesure que les intellectuels marxistes rompaient avec les partis communistes et surtout avec le modèle de société socialiste jusqu'alors offert à leur admiration, la pensée marxiste s'est éloignée encore plus des pratiques sociales et s'est voulue pure théorie. En fait, elle s'est épuisée dans plusieurs directions. Ses produits intellectuellement les plus brillants sont apparus lorsqu'elle se dissociait complètement de tout effort d'analyse économique et sociale, lorsqu'elle ne parlait plus ni de la domination capitaliste ni de la lutte des classes mais lorsqu'elle s'efforçait de retrouver les principes fondamentaux de la [178] pensée de Marx. Ce que fit Althusser en construisant une œuvre de grande qualité et dont la solidité même semble construire une muraille entre la pensée marxiste et l'analyse des faits sociaux. D'autres au contraire s'efforcèrent de chercher des compromis entre cette pensée marxiste pure et les réalités sociales et politiques dans lesquelles ils cherchaient à l'appliquer. Ce qui donna naissance à une nouvelle génération de penseurs social-démocrates comme Milliband ou Poulantzas, malgré les petites différences qui les séparent. Cette catégorie de marxistes, comme les théologiens catholiques de la fin du XIX ${ }^{\mathrm{e}}$ siècle, s'efforcèrent d'assouplir les principes de la doctrine et nous rappelèrent par exemple que si l'État est l'agent de la classe dirigeante, il n'en a pas moins une certaine autonomie, type de formule qui a l'avantage de pouvoir dire n'importe quoi. À un niveau moins élevé le marxisme servit, surtout en France, à manifester le malaise d'une population universitaire éloignée de la société et de l'ensemble de ses forces so- 
ciales actives. Au moment où les thèmes nés en Mai 1968 se développent presque partout sauf dans l'université, celle-ci, ayant abandonné l'inspiration de Mai, semble n'en retenir que le discours, dans ce qu'il a de plus archaïque et de plus théologal. Ce marxisme intellectuel, tout comme le vocabulaire des partis communistes, est devenu un simple signe de reconnaissance. Que quelqu'un dise l'Italie ou la formation sociale italienne ne fait pas grande différence, mais permet, dans le second cas au moins, d'identifier l'idéologie de celui qui parle, ce qui est important dans une phase de refus et surtout de dissociation entre la pratique et la théorie intellectuelle. Mon éloignement du marxisme, qui n'est pas séparable de ma critique de tout l'objectivisme de la pensée du siècle passé est évidemment renforcé par la décadence de la pensée marxiste elle-même. Mais depuis 1975 la crise du marxisme ne peut plus être réduite à sa stérilité. L'apparition d'un parti communiste hyperléniniste au Portugal, les révélations de Soljenitsyne et la montée du grand mouvement des dissidents en Union soviétique et plus récemment enfin la rupture en France entre le parti communiste et ses derniers intellectuels, tout cela a enlevé à la pensée marxiste ses garants politiques et historiques. On n'était pas marxiste comme on était platonicien. Un marxiste parlait toujours plus ou moins au nom de la classe ouvrière ou au nom du bloc socialiste, plus récemment au nom des mouvements de libération du Tiers Monde. Or tous ces principes de légitimité ont disparu. L'image de l'Union soviétique est aujourd'hui d'abord celle d'un régime totalitaire et répressif ; les mouvements de libération du Tiers-Monde ont été remplacés dans beaucoup de cas par des dictatures qui, même quand elles sont modernisatrices, ne sont plus qualifiées pour parler au nom des peuples; enfin la pratique syndicale est presque partout très éloignée des thèmes révolutionnaires du tournant du siècle. La destruction de ces garants politiques et historiques d'une pensée sociale explique le violent mouvement de profanation dont le communisme et le marxisme sont aujourd'hui les victimes. Parce qu'ils ont invoqué des dieux, ils suscitent la violence des apostats. Cette réaction n'est pas seulement celle de quelques " nouveaux philosophes ». Le recul de la croyance marxiste est général. Il laisse la place en Europe à une nouvelle forme de pensée libérale dont l'expression principale est produite, par un étrange retournement historique, par ceux qui se 
nomment eux-mêmes marxistes dans les pays communistes et qui défendent les droits de l'homme et du citoyen contre l'État autocratique ou totalitaire. Cette poussée [179] libérale, qui se tourne d'abord contre l'Union soviétique mais aussi contre le parti communiste, dont les formes d'organisation et d'expression, bien qu'elles n'aient guère changé, deviennent tout d'un coup insupportables, dominent le moment intellectuel dans lequel nous réfléchissons. C'est pourquoi je tiens à définir ma démarche, si profondément opposée qu'elle soit au marxisme, comme un post-marxisme et à l'opposer profondément à une démarche libérale avec laquelle cependant elle doit établir certaines relations. Je suis post-marxiste en ce sens que j'essaye de construire une démarche d'analyse qui occupe dans notre société, société d'action et non d'évolution, une place analogue à celle qu'occupa la pensée marxiste dans la société industrielle. Je pense même que c'est seulement aujourd'hui que des idées, déjà en germe dans la pensée marxiste, comme la lutte des classes et les mouvements sociaux, peuvent enfin recevoir leur véritable importance, car de telles idées s'incorporent naturellement dans une sociologie de l'action et ne peuvent pas avoir d'importance centrale dans une économie sociale des contradictions internes du capitalisme et des lois historiques d'aggravation de ces contradictions. Je m'oppose dans nos sociétés à une pensée essentiellement libérale parce que celle-ci s'oppose à l'État et que dans notre partie du monde la société n'étant pas entièrement soumise à l'État les problèmes sociaux sont dominés par le conflit des classes et non pas par la contradiction de l'État et du citoyen. Mais je ne rejette pas entièrement la pensée libérale car j'ai dit que les mouvements sociaux ne sont que le point d'équilibre entre une intervention institutionnelle et une action critique, entre la participation au système politique et l'attaque contre l'État. Notre pensée a été dominée depuis 1905 par le modèle révolutionnaire, par l'association d'un mouvement social et de la crise violente d'un État. Nous vivons en fait dans des conditions historiques où les mouvements sociaux se trouvent davantage associés à un processus démocratique. C'est donc de là que nous devons partir, sans pourtant oublier, que cette situation est minoritaire à l'échelle du monde et que l'association des problèmes sociaux et des problèmes de l'État commande dans la plupart des pays l'action politique. Enfin je crois dangereux de retarder l'indispensable recherche des nou- 
veaux mouvements sociaux. Je ne me laisse pas convaincre par ceux qui croient au remplacement des conflits de classe fondamentaux par des tensions multiples liées à des processus constants et complexes de changement social. Non tout n'est pas changement; non les problèmes de la domination sociale ne sont pas remplacés par ceux de la conduite des transformations historiques. Toute la sociologie ne se réduit pas à la sociologie du développement et nous découvrirons vite, dans les années qui viennent, l'importance primordiale pour nous de problèmes qui ne peuvent être compris que comme la manifestation des conflits sociaux et des orientations culturelles propres à la société programmée.

Ayant ainsi défini une position par rapport aux problèmes sociaux de la société où je vis, je dois évoquer ce qui caractérise d'autres modes de développement ou au moins un d'entre eux. On peut regrouper de manière simple les modes de développement en cinq types, situés symétriquement par rapport à un mode central, celui des pays capitalistes dits centraux dont l'importance historique a été exceptionnelle à cause de l'hégémonie qu'il a exercé sur la plus grande : partie du monde. Dans ce type qu'on peut nommer libéral c'est une bourgeoisie nationale qui a l'initiative du développement économique et l'État [180] national n'est à l'intérieur pour l'essentiel que son agent, tout en menant à l'extérieur une action proprement étatique et conquérante. Si on s'éloigne de ce type central en direction des pays dont la bourgeoisie nationale était plus faible, on rencontre d'abord un ensemble de pays où l'État se donna pour tâche principale de constituer un capitalisme national. Tel fut le cas de l'Allemagne, de l'Italie et du Japon et ce n'est pas un hasard si c'est dans ces trois pays que, sous des formes extrêmement diverses, la crise du capitalisme donna naissance à des régimes nationalistes, impérialistes et pour certains d'entre eux fascistes. Plus loin encore des pays centraux se rencontrent les pays où l'État national était surtout le garant des privilèges d'une classe dominante décadente. Dans ces pays, la pénétration du capitalisme occidental provoqua plus encore que des crises sociales une crise de l'État et la prise du pouvoir le plus souvent par des partis communistes. Un autre ensemble de modes de production s'écarte du modèle central dans une direction opposée. Ce n'est plus ici l'État national autocratique qui domine une bourgeoisie 
nationale faible ou corrompue, c'est une bourgeoisie étrangère qui se substitue à la bourgeoisie nationale, ce qui est le cas des sociétés dépendantes. C'est même souvent, comme dans les sociétés coloniales, une domination extérieure à la fois économique, administrative et militaire qui se substitue à la bourgeoisie et à l'État national. C'est seulement aux sociétés dépendantes que je veux m'intéresser ici et plutôt à titre de simple introduction ou commentaire aux remarques faites par plusieurs participants de ce colloque sur la situation québécoise car le Québec n'appartient certainement ni aux pays colonisés ni aux sociétés dominées par des États autocratiques ou des États fortement industrialisateurs, de type bismarckien. Rappelons brièvement la caractéristique principale des sociétés dépendantes. Je les ai nommées désarticulées, c'est-à-dire que leur organisation économique et leur organisation sociale sont séparées du fait même que le pouvoir économique est entre les mains d'étrangers et du système capitaliste international. Dans les sociétés dépendantes les classes supérieures sont plus dominantes que dirigeantes et surtout les classes d'appui, ce qu'on peut nommer vaguement la petite bourgeoisie, est plus influente que la classe supérieure elle-même, la grande bourgeoisie. Cette distance entre le pouvoir externe de production et le pouvoir interne de reproduction donne une extrême autonomie à l'espace politique. Alors qu'on est tenté de croire que la domination économique externe réduit cette autonomie, elle l'élargit au contraire. La plupart des pays dépendants possèdent un système politique très actif et très autonome. Plus autonome encore, est, au cœur de cet espace politique, la vie culturelle ou intellectuelle, ce qui se manifeste souvent par le rôle politique et social important des universités et surtout par la dissociation, parfois spectaculaire des idées et des conduites politiques des étudiants et des intellectuels et de ceux du reste de la société. Les exemples du Pérou, de l'Argentine, ou du Vénézuela, si différents qu'ils soient les uns des autres, ont montré de manière spectaculaire l'importance de cette autonomie universitaire.

L'autonomie du champ politique signifie aussi que des forces politiques et même des États interviennent comme les agents principaux de ces mouvements de développement, au point que parfois on pourrait parler de mouvements étatiques. Les mouvements 
sociaux dans les sociétés dépendantes au lieu d'être directement les agents d'expression d'un problème ou d'un processus, sont des [181 réponses à ce qu'on peut nommer des modes d'intervention étatique. Ceux-ci doivent être définis par l'action de l'État, agent de transformation historique, dans une situation définie par le croisement des deux grands axes de l'analyse sociologique. L'État qui se situe dans l'angle formé par le croisement de l'action historique et du changement peut être nommé l'État national populaire. Il est le principal mode d'intervention étatique dans les sociétés dépendantes, sociétés entraînées dans le changement et qui se définissent par leur capacité d'action plutôt que par leur situation de crise. À l'inverse, l'intervention étatique qui répond à un ordre despotique et à une société en crise est l'État communiste. Entre ces deux modes principaux et opposés d'intervention étatique, se placent deux autres. D'abord l'État social démocrate qui intervient dans les sociétés marquées par l'action plus que par la crise mais aussi par un ordre social conservateur plutôt que par le changement. Enfin l'État de socialisme communautaire est celui qui joue un rôle libérateur de changement mais dans une société en crise. Ces quatre types correspondent donc aux quatre combinaisons de l'axe : action-crise et de l'axe : ordre-changement. Ces modes d'intervention étatique sont instables parce que l'État y joue : un double rôle : d'un côté un rôle étatique et de l'autre un rôle social, c'est-à-dire en fait d'agents ou de substituts d'une classe dirigeante. Chacun des quatre modes d'intervention étatique qui viennent d'être distingués tend ainsi constamment à éclater. L'État national populaire tend à devenir soit une dictature militaire soit l'agent d'un capitalisme d'État périphérique. L'État de socialisme communautaire, tel qu'on le trouve souvent en Afrique, peut se transformer en dictature patrimoniale ou au contraire en support étatique du néo-colonialisme. L'État communiste tend à se transformer en dictature idéocratique, soit en technocratie d'État. Enfin l'interventionnisme de l'État social-démoerate peut laisser la place à un social-capitalisme, comme dans le cas allemand contemporain mais a pu aussi, surtout dans le passé, se renverser en État fasciste. Ces indications beaucoup trop brèves n'ont d'autre but ici que d'indiquer ce que devrait être l'analyse des luttes historiques comme réponse aux modes d'intervention étatique et à leurs formes de décomposition. Ces luttes historiques peuvent d'abord apparaître comme le prolongement du mode d'intervention étatique au point même de sembler être le résultat d'une mobilisation sociale dirigée par 
l'État. Tel est le cas du populisme dans les sociétés dépendantes, qui apparaît d'abord comme le produit de l'intervention de l'État national populaire. Mais cette interprétation est insuffisante et il est nécessaire d'analyser le populisme en lui-même. Ce que j'ai plusieurs fois tenté de faire en montrant qu'il combine trois orientations différentes : une action de classe, ce qui rappelle que dans tout processus de développement existent aussi des problèmes structurels ; une lutte nationale menée contre la dépendance externe ; enfin une action modernisatrice, une volonté de développement. Ce populisme est aussi instable que le mode d'intervention étatique auquel il correspond et il tend à se décomposer de la même manière que lui. Si l'État national populaire se transforme en dictature militaire, le populisme peut se décomposer au profit d'un nationalisme révolutionnaire. Si au contraire l'État national populaire se transforme en capitalisme d'État périphérique, le populisme cède la place à une action de classe révolutionnaire. Des analyses semblables peuvent être menées dans le domaine des autres modes d'intervention étatique. La lutte [182] historique qui correspond à l'État communiste est l'action menée par un parti de libération sociale et nationale. action dont le parti communiste chinois ou celui du Viet-Nam ont donné les exemples les plus importants ; mais lorsque l'État communiste se transforme en dictature idéocratique il suscite un mouvement anti-étatique de démocratisation ; là au contraire où l'État communiste devient une technocratie il rencontre en face de lui un mouvement de défense populaire. On reconnaît ici les deux tendances opposées mais inséparables de la dissidence soviétique. Sakharov est la figure de proue du mouvement pour la démocratisation tandis que Soljenitsyne est l'image même d'une défense populaire et par moment presque slavophile contre la technocratie d'État. Le socialisme communautaire suscite une mobilisation communautaire qui va se dégrader en défense technique lorsque l'État devient une dictature patrimoniale ou se transformer en action de classe, menée surtout par une contreélite, lorsque l'État devient un agent du néo-colonialisme. Enfin le travaillisme, lutte historique qui correspond au mode social démocrate d'intervention étatique se transforme en anti-fascisme lorsque l'État devient fasciste et en mouvements pour l'autoorganisation lorsque la social-démocratie devient un socialcapitalisme, comme le montre le développement des comités de citoyens aussi bien en Allemagne ou en Norvège qu'au Canada. 
Les analyses menées sur les luttes historiques dans les sociétés dépendantes devraient être généralisées et appliquées à tous les types qui viennent d'être construits. Chacun en effet est une réalité mixte dans laquelle se combinent une dimension de classe, une dimension nationaliste et une dimension modernisatrice.

Surtout Robert Vandycke a raison de rappeler que toute lutte historique est associée à des conduites de crise, liées à la rupture d'un ordre, à la désorganisation sociale et au changement luimême. Le mouvement de l'histoire trouble l'agencement du système social comme l'État introduit dans le changement historique une image déformée de l'ordre et même des rapports de classes et de l'historicité. Ce qui montre bien que les phénomènes historiques n'ont pas la pureté des catégories de l'analyse sociologique.

Ces observations très générales pourraient servir d'introduction à une étude des luttes historiques au Québec. Plusieurs de leurs aspects ont été traités par Jacques Dofny, Gabriel Gagnon, Paul Bélanger et Céline Saint-Pierre. Je me contenterai ici de poser un principe d'analyse. Le niveau de développement économique, de modernisation et de richesse du Québec est évidemment très éloigné de celui des pays dépendants, mais ceci ne constitue d'aucune manière un obstacle à l'application au Québec des principes d'analyse valables pour l'ensemble des sociétés dépendantes. Il y a d'ailleurs plus de distance encore entre l'Argentine et la Bolivie qu'entre le Québec et l'Argentine, même en tenant compte du recul spectaculaire de ce dernier pays. La similitude des situations se marque d'abord par l'extrême autonomie de l'espace politique et par l'autonomie plus grande encore de l'espace culturel au Québec. Le rôle des universités ou de Radio-Canada dans la prise de conscience nationale moderne est assez semblable à celui qu'ont joué les grandes universités dans beaucoup de pays latino-américains au cours des cinquante dernières années. Parallèlement, la sociographie du Québec a toujours insisté sur le rôle de la petite bourgeoisie dissociée des propriétaires principaux, des moyens de production et aussi [183] sur l'importance des catégories définies davantage par leur place dans le changement social que par leur position de classe. Mais l'essentiel est surtout de rappeler que les trois composantes des luttes historiques : classe, nation et modernisation se croisent de manière encore plus complexe dans un pays hautement développé 
comme le Québec que dans les autres sociétés dépendantes. L'importance de l'industrialisation du Québec a entraîné celle du mouvement syndical et celui-ci ne s'est jamais confondu simplement avec le nationalisme. Les efforts pour unir revendications nationales et revendications sociales sont constants et aboutissent toujours à des demi-échecs. Enfin, la modernité du Québec interfère directement avec la force de la revendication nationale et avec l'activité des luttes sociales et des négociations collectives.

Paul Bélanger et Céline Saint-Pierre soulignent fortement qu'on ne peut réduire l'étude de la situation québécoise ni à une lutte nationale ni à une lutte sociale, mais je suis peut-être plus convaincu qu'eux de la nécessité de reconnaître : le croisement des trois principes d'analyse que je viens d'indiquer. Ce qui donne toute son importance à l'idée de double stratification défendue par Jacques Dofny. Cette absence d'intégration est caractéristique des sociétés dépendantes; elle est plus nette encore au Québec. Le Québec est plus moderne que les autres pays dépendants ; l'action de classe y est plus développée et sa base de défense nationale est aussi plus forte puisqu'une culture francophone qui n'est plus maintenue naturellement par une forte poussée démographique ou un solide encadrement religieux doit se défendre de manière de plus en plus activ4 non seulement contre la domination anglophone environnante, mais aussi contre l'appui important qu'apporte à celle-ci les néo-canadiens. De là l'extrême coml1lexité de la politique québécoise qui va bien au-delà de l'autonomie des revendications sociales et des revendications nationales. Il semble que ce pays soit entraîné simultanément dans trois directions définies chacune par la dominance d'une des composantes des luttes historiques. D'un côté c'est l'incorporation du Québec à l'espace économique nord américain qui semble l'emporter et c'est à l'intérieur de cette incorporation que les syndicats liés à la centrale américaine apportent leur appui à un gouvernement qui insiste plus sur la défense d'une culture et de catégories moyennes que sur des transformations économiques et sociales fondamentales; gouvernement qui pour être indépendantiste ne tire pourtant pas sa force principale d'un mouvement proprement indépendant. D'un autre côté s'aperçoit une lutte qui s'organise autour du thème national et anti-impérialiste, luttes qui se rapprochent soit d'un populisme révolutionnaire soit d'un nationalisme révolutionnaire, particulièrement influent dans des milieux universitaires et étudiants. En- 
fin, a toujours existé au Québec une tendance politique qui correspond surtout à l'appartenance du Québec, rappelée ici par Gabriel Gagnon, à la modernité nord-américaine et qui peut prendre la forme particulière d'une hétéronomie culturelle ou politique, correspondant à la situation économique d'une région qui a été incorporée à un marché et à une production très modernes plutôt qu'elle n'a créé son propre pôle de développement.

Pas plus au Québec qu'ailleurs il n'est possible d'unifier ces trois tendances fondamentales et pas davantage de choisir l'une d'elles contre les autres. De la même manière, Allende au Chili ne pouvait pas choisir entre l'action de classe [184] représentée surtout par la centrale syndicale, l'action modernisatrice à laquelle étaient sensibles des catégories moyennes et populaires et le courant antiimpérialiste qui dominait le parti socialiste lui-même. Au Québec aussi existent des incohérences par exemple entre le vote provincial et le vote fédéral et une instabilité qui modifie fréquemment les relations entre les syndicats et les forces politiques mais cette instabilité et cette complexité ne conduisent pas nécessairement à la décomposition de la lutte historique. Le Québec peut aussi bien suivre une voie mexicaine, c'est-à-dire le développement d'un État national combinant en lui les tendances qui viennent d'être distinguées et limitant leurs fluctuations à l'intérieur d'un contrôle politique étroit. Ce qui revient à dire que le seul instrument d'intégration des trois dimensions de la situation québécoise est l'indépendance, mais celle-ci ne peut être atteinte que si intervient un facteur extérieur aux luttes sociales et nationales qui ne peuvent pas se combiner spontanément. Ce facteur externe ne peut pas être d'ordre militaire et géo-politique ; il a peu de chance d'être économique. Il ne peut donc être que culturel. Le Québec semble entraîné de plus en plus nettement vers un choix vital entre l'indépendance et la disparition, c'est-à-dire l'incorporation à l'espace politique et culturel nord-américain. 


\section{CONCLUSION EN FORME D'INTRODUCTION}

$\underline{\text { Retour à la table des matières }}$

L'ensemble des idées présentées et discutées au cours de ce colloque et en particulier celles que j'ai exposées moi-même risque à chaque instant de n'être qu'un corps d'opinions dont l'apparence d'objectivité tient simplement à ce qu'elles ne sont reliées que de manière très indirecte à des idéologies, c'est-à-dire à la manière dont les acteurs sociaux se représentent les situations où ils sont engagés. Il ne faut donc considérer ces idées que comme des éléments qui doivent permettre d'élaborer des recherches. Mais cette expression doit être prise en un sens exigeant. Après s'être demandé, en sociologue ce qu'il faut penser, il faut maintenant poser la question : que faut-il faire ? Une démarche intellectuelle n'a d'intérêt que pour autant qu'elle produit une méthode de recherche spécifique. Il ne semble pas que les méthodes habituelles des sciences sociales correspondent aux besoins d'une sociologie de l'action. L'enquête sociologique saisit surtout des comportements de consommation ou des dispositions à faire des choix dont les termes sont pré-déterminés par les catégories de la pratique sociale. C'est là un domaine important d'étude et qui a le grand avantage d'apporter des informations dans les termes qui sont ceux mêmes des décisions que doivent prendre les acteurs. Chacun d'entre nous, pendant une campagne électorale, s'intéresse à connaître les intentions de vote de diverses catégories sociales, des hommes et des femmes, des patrons et des ouvriers, des jeunes et des vieux, des citadins et des ruraux. Mais rien n'est plus éloigné que cette méthode d'étude de la consommation d'une étude de la production de la société par elle-même. L'enquête qui place l'acteur devant une situation ne peut pas être utilisée lorsqu'il s'agit de comprendre comment les rapports sociaux construisent la situation. On est tenté alors de recourir à la méthode la plus éloignée de l'enquête sociologique, celle qui est le plus couramment utilisée par les historiens : l'examen des documents, le plus souvent écrits, qui [185] nous informent à la fois sur les intentions des acteurs collec- 
tifs, sur leurs conflits et leurs négociations et sur les formes de domination sociale qui orientent l'activité des organisations et des institutions. Malheureusement cette méthode est aussi inadaptée que la première à l'objet que nous considérons. Comme il est naturel, l'étude des documents joue un rôle essentiel dans la connaissance du changement social mais l'action sociale elle-même produit peu de documents et ceux qu'elle produit sont soit des documents idéologiques soit des documents proprement historiques c'est-à-dire dans lesquels plusieurs significations se trouvent mélangées dans une conjoncture donnée. Il faut donc inventer une nouvelle démarche méthodologique. Je l'appelle l'intervention sociologique. Elle repose sur trois principes élémentaires. Le premier est que l'action collective doit être saisie directement, c'est-à-dire comme action d'une collectivité ou d'un groupe placé en relation avec des partenaires ou adversaires sociaux dans un cadre historique déterminé. L'enquête individuelle ou même de groupe doit être remplacée par l'étude de collectifs militants, d'acteurs collectifs qui sont non seulement étudiés dans leur action collective, mais qui acceptent de participer à l'étude en tant que militants et parce que la recherche leur semble utile du point de vue même de leur action militante. Le deuxième principe est que la recherche, quand elle porte sur des acteurs et surtout sur des mouvements sociaux qui sont les acteurs de niveau le plus élevé, doit porter directement sur le sens que les acteurs donnent à leur conduite. L'objet de l'analyse sociologique doit être l'analyse que les acteurs mènent d'eux-mêmes, de leur action et de leur situation. L'intervention sociologique est l'analyse d'une autoanalyse. Le troisième principe, le plus difficile à élaborer et à respecter, est que le chercheur qui cherche à connaître l'action et qui s'adresse à des militants en tant que tels ne peut pas lui-même se placer en situation d'observation neutre, comme s'il était un entomologiste, puisqu'une telle attitude aboutirait nécessairement à détruire l'objet de l'étude. Comment se délivrer de cette attitude sans pour autant devenir l'idéologue du groupe qui s'identifierait à lui, l'aiderait à formuler sa représentation de lui-même, de son adversaire et de l'enjeu de leur conflit mais qui détruirait ainsi son rôle de sociologue puisque la sociologie explique l'acteur par le rapport où il est placé au lieu d'interpréter ce rapport du point de vue d'un des acteurs ? La solution est que le chercheur, qui observe non pas un mouvement mais une lutte concrète dans laquelle se mélangent diverses significations, doit se placer du point de vue du mouvement social qu'il suppose présent 
dans la lutte. Il parle donc au nom de la signification la plus élevée de la lutte de sorte que sa relation avec le groupe étudié représente la relation de la lutte au mouvement présent en elle mais mêlé à d'autres types de conduites collectives. La combinaison de ces principes a conduit à construire des interventions sociologiques par lesquelles les chercheurs travaillent avec des groupes d'acteurs, qui continuent à se comporter au cours de la recherche en tant que militants réfléchissant sur leur propre action. Ces groupes sont d'abord confrontés à leurs partenaires réels puis ils sont invités à mener l'analyse de leurs conduites et de leurs déclarations, telles qu'elles ont été indiquées dans ces rencontres. C'est ensuite seulement que le chercheur s'efforce de distinguer les diverses significations de cette action en introduisant dans le groupe l'image du mouvement social qu'il croit impliqué dans cette lutte particulière. L'ensemble d'une intervention, telle que je viens de la résumer très [186] brièvement ici, représente une centaine d'heures de travail du chercheur avec chaque groupe d'une dizaine de personnes mais l'intervention elle-même ne constitue que la phase centrale d'une recherche. Avant elle, la connaissance directe du mouvement grâce à des documents écrits ou oraux permet au chercheur d'orienter ses hypothèses. Après l'intervention doit se développer ce que je nomme sociologie permanente c'est-à-dire que les acteurs doivent se ré approprier les résultats de l'intervention dans leur propre perspective et les chercheurs observer les effets de l'analyse sur l'action, acteurs et chercheurs entrant en dialogue comme, d'une manière entièrement différente, acteurs et analystes de la vie économique. Les résultats de l'intervention seront renforcés par cet examen prolongé de leur capacité de rendre intelligible une lutte, son déroulement et ses résultats, au delà des intentions de ses dirigeants. Je rejoins ici Gabriel Gagnon tout en soulignant que l'analyse ne peut pas être validée par sa convergence avec l'idéologie des acteurs. Si on croit que les mouvements sociaux sont les acteurs principaux de la société, c'est bien au niveau de leur capacité d'agir que doit se situer l'intervention du sociologue. Celle-ci n'a rien à voir avec une action de propagande ou de formulation idéologique ; elle n'apporte pas davantage de solution aux problèmes sociaux posés mais elle peut contribuer à augmenter la capacité d'action des acteurs. Le sociologue qui s'engage dans de telles interventions doit être convaincu de leur caractère bénéfique. Celui-ci tient à ce que l'intervention renforce l'ac- 
tion contre l'ordre, la production contre la reproduction, les rapports sociaux et leurs enjeux contre les conduites d'ordre et de crise. La sociologie n'a pas atteint un degré de formalisation tel que la connaissance puisse être ici sa propre fin. Ceux qui ne parlent que de sociologie pure ou désintéressée ne sont en fait que des observateurs qui expriment des opinions elles-mêmes teintées d'une idéologie assez vague pour ne pas pouvoir être facilement identifiée. L'intervention sociologique, la création d'une pratique sociologique, est au contraire la condition, non pas de la subordination de la recherche aux pouvoirs établis mais de l'analyse critique des pratiques sociales et de la remontée à partir des objets vers les rapports sociaux. Plus est réussie cette remontée, plus est augmentée la capacité d'action et d'analyse d'une société sur elle-même et plus reculent les risques de violence, qu'il s'agisse de la violence étatique ou de la violence liée à la décomposition des rapports sociaux, de la violence d'ordre ou de la violence de crise. La science sociale n'a plus pour objet principal d'étudier la nature de la société mais les mécanismes de l'action sociale. L'intervention sociologique est la méthode qui correspond à cet objectif. Il est préférable dans un premier temps qu'elle soit appliquée d'abord à l'étude des actions collectives de niveau le plus élevé, aux mouvements sociaux, qu'il s'agisse de mouvements populaires ou de mouvements de classes dirigeantes. Mais ensuite il faudra qu'elle redescende progressivement vers d'autres domaines de la vie sociale, non pas pour les étudier dans leur intégralité mais pour y retrouver la trace, de plus en plus indistincte, de l'action sociale et des systèmes d'action. Partie des mouvements sociaux, elle descendra d'abord vers les pressions institutionnelles et les revendications organisationnelles avant de passer à l'étude des conduites que l'ordre définit comme délinquantes ou marginales puis à l'étude des conduites collectives de crise et enfin à l'étude des conduites de changement, d'innovation et de libération ou de résistance à ces changements.

[187]

Je souhaite que l'ensemble des réflexions et des discussions de ce colloque soit considéré par tous ses participants comme une introduction à de nouvelles recherches, à la création de nouvelles pratiques sociologiques qui permettront à. la fois le développement de la connaissance et la défense des libertés et de la démocratie. 


\section{RÉSUMÉ}

\section{$\underline{\text { Retour à la table des matières }}$}

Ce texte converge vers la proposition principale que l'objet central de l'analyse sociologique est bien celui des relations entre la synchronie et la diachronie, entre les modes de production sociale des types sociétaux et les modes de développement de sociétés particulières. Aussi l'auteur présente-t-il d'abord différents concepts relatifs aux systèmes d'action sociale et à leurs composantes : l'historicité, fondement même des rapports de classes, les systèmes politico-institutionnel et organisationnel. D'autres concepts lui permettent de repérer les régions du fonctionnement d'une société : les théories régionales de la reproduction, de la crise et du changement complètent alors celle des systèmes d'action sociale. Cet ensemble de concepts produit une analyse structurale-synchronique des types généraux de sociétés, en tant que niveaux d'une historicité ouverte sur différentes formes de mouvements sociaux et de rapports de classes. S'attardant ensuite à la question du changement, l'auteur en analyse les facteurs exogènes à une société particulière avant d'aborder la question de l'État, agent de rapports intersociaux mais aussi de changement et de transformation de même que d'ordre et de reproduction. Il soutient finalement que c'est l'analyse des rapports de classes, parce qu'ils sont justement tout à la fois rapports de production et de reproduction, qui permet de croiser l'analyse de la structure et du changement, celle des classes dirigeantes d'une société et de l'État : l'auteur voit une application possible de cette théorie aux sociétés dépendantes, au Québec en particulier, où actions de classe, lutte contre la domination nationale et conflits amenés par la modernisation apparaissent désarticulés. 


\section{SUMMARY}

\section{$\underline{\text { Retour à la table des matières }}$}

The author's principal proposition is that the central objective of sociological analysis is the relationships between synchrony and diachrony, between modes of social production of societal types and modes of development of particular societies. In this perspective the author presents various concepts relative to social action systems and to their components : historicity, in itself the basis of class relations, and politico-institutional and organizational systems. Others concepts are introduced to permit the author to identify the functioning areas of a society : regional theories of reproduction, of crisis and of change complement the theory of social action systems. This set of concepts produces a structural synchronic analysis of the general types of societies, seen as levels within an historical analysis including various forms of social movements and class relations. The author then studies the question of change, analyzing exogenous factors in a particular society, before attacking the question of the State as agent of intersocial relations, as well as of change and transformation and of order and reproduction. Finally, he asserts that it is the analysis of c1ass relations, precisely because they are at once relations of production and of reproduction, which pennits us to bring together the analysis of structure and of change in the ruling classes of a society and of the State. Touraine sees a possible application of this theory in dependent societies, Quebec in particular, where class actions, struggle against national domination and conflicts brought on by modemization appear dislocated.

\section{RESUMEN}

Este texto converge hacia la proposición principal que el objeto central deI análisis sociológico es bién el de la relaciones entre la sincronía y la diacronía, entre los modos de producción social de los tipos societales y los modos de desarrollo de sociedades particulares. El 
autor presenta al comienzo diferentes conceptos relativos a los sistemas de acción social y a sus componentes : la historicidad, base misma de las relaciones de clases, los sistemas politico-institucionales y organizacionales. [188] Otros conceptos le permiten descubrir las regiones dei funcionamiento de una sociedad : las teorías regionales de la reproducción, de las crises y del cambio completan de esta manera la teoria de los sistemas de acción social. Este conjunto de conceptos produce un análisis estructural-sincrónico de los tipos generales de sociedades, como niveles de una historicidad abierta sobre diferentes formas de movimientos sociales y de relaciones de clases. Enseguida el autor analiza la cuestión del cambio y de los factores exogenos a una sociedad particular antes de abordar la cuestión del Estado, agente de relaciones intersociales pero también de cambio y de transformación asi mismo que de orden y ve reproduccicin. Sostiene finalmente que es el analisis de la relaciones de clases, porque son justamente, todo al mismo tiempo relaciones de producción y de reproducción, que permite el cruzamiento deI análisis de la estructura y deI cambio, el de las c1ases dirigentes de una sociedad y deI Estado: Touraine ve una aplicación posible de esta teoría a las sociedades dependientes, a Quebec en particular, donde acciones de clases, lucha contra la dominación nacional y conftictos consecuentes a la modernisación aparecen desarticulados.

\section{Fin du texte}

